\title{
Element Desorption from and Crystal Dissolution of a Smectite by Interaction with the Bacteria Escherichia coli and/or Varied Amino Acids
}

\author{
K Semhi ${ }^{1}$, N Clauer ${ }^{2 *}$, A Sayeigh ${ }^{3}$ and S Bani ${ }^{1}$ \\ ${ }^{1}$ Sultan Qaboos University, Oman \\ ${ }^{2}$ Institut de Physique du Globe de Strasbourg (UdS-CNRS), France \\ ${ }^{3}$ Ministry of Municipality Affairs and Urban Planning, Bahrain
}

Submission: November 27, 2019; Published: December 16, 2019

*Corresponding author: N Clauer, Institut de Physique du Globe de Strasbourg (UdS-CNRS), 1 rue Blessig, 67084 Strasbourg, France

Abstract

Three-week experiments were completed to evaluate the interaction of the bacteria Escherichia coli and amino acids (glutamic acid, alanine, arginine, serine and leucine) on the dissolution and/or desorption rates of a smectite-type clay mineral. A systematic dominant leaching of $\mathrm{Na}$ confirms that the initial smectite was Na-enriched. The bacteria Escherichia coli induced leaching of a Si/Al ratio far from that released by either the amino acids or by a combination of the bacteria and amino acids. Conversely, this ratio is close to the value of the smectite itself. Therefore, the bacteria has a probable dissolution impact rather than a desorption action. This preferential action is indirectly corroborated by a low removal of Na by the bacteria. Removal impact of the bacteria on the smectite decreased when mixed with any of the amino acid. The highest releases of $\mathrm{Si}, \mathrm{Al}, \mathrm{Mn}$ and Fe characterize batches without amino acids, while most released $\mathrm{Ca}$ and $\mathrm{K}$ occurred with the amino acids. Potassium, $\mathrm{P}, \mathrm{Mg}, \mathrm{Na}$ and $\mathrm{Ca}$ are more released into solutions containing several amino acids, while $\mathrm{Fe}$ is preferentially leached by solutions with only one amino acid.

This study highlights also the potential roles of amino acids in soils and other surficial geological systems. The distribution patterns of the leached rare-earth elements (REEs) confirm a clear distinction between the impact of the bacteria that produces a progressively decreasing contents of the light REEs and a flat distribution for the heavy REEs and those, more variable, of the amino acids. The impact of the amino acids influences the REE distributions, probably along complexation processes due to strong metal sorption on oxides by organic complexes.

Keywords: Smectite; Bacteria Escherichia coli; Amino acids; Mineral dissolution; Elemental desorption; Cationic exchanges; Complexation

\section{Introduction}

Many studies were already designed for the calculation of mineral dissolution rates [1-3] that are often dependent on the $\mathrm{pH}$ of the interacting fluids that control adsorption/desorption and ionic exchange reactions of the selected minerals. The removed elements increase generally with the $\mathrm{pH}$ conditions and/or the temperature $[4,5]$. Numerous experiments include calculations of dissolution rates of clay minerals based on their well-known exchange capacities, while organic ligands of soils and the associated fluids are known to affect the reaction rates of any mineral by changing its adsorption and desorption potentials [6]. In turn, the acidity of soils induced by such organic compounds reduces the microbial activity $[7,8]$ showed that amino acids such as histidine, lysine and arginine interact more efficiently with the negatively charged surface of amorphous silica than other non-basic amino acids because of a greater dissociation, thus forming cationic species. The same authors and Kawano et al. [10] showed that cysteine, asparagine, serine, tryptophan, alanine and threonine also enhance the dissolution of amorphous silica.

Amino acids are organic compounds that yield $\mathrm{NH}_{2}$ and $\mathrm{COOH}$ functional groups, as well as side chains that are specific to each type. Important in the nutrition cycle due to their biological significance as protein carriers, these amino acids contribute to a number of processes as bio-carriers and bio-synthesizers. Because of that biological significance, amino acids are important components along the nutrition process; they are commonly used, for instance, in nutritional complements, in food technology, as well as in fertilizers. Beyond potentials in mineral dissolution, organic complexes influence also strongly the sorption potentials 
of metallic elements at the surface of clay crystals in sub-surface environments, especially because of their functional groups $[11,12]$. In this overall context, we completed varied leaching and dissolution experiments of a smectite-type bentonite in presence of one or several amino acids, the purpose being rather to evaluate their individual and collective efficiency than producing more values of the sole rates. As microbes can also mobilize metals and attack mineral surfaces especially by redox processes [13-17], the impact of bacteria Escherichia coli (labeled as E. coli along the text) has also been examined for comparison.

\section{Material and Methods}

Smectite, the selected clay mineral, has been extracted from a bentonite that is a rock facies used extensively in industry because of its high adsorption and desorption capacities. The mineral separate used for the experiment is pure with all X-ray diffraction harmonic 001 peaks of the untreated, ethyleneglycolated and heated fraction (Figure 1). The experience consisted in an interaction of $1 \mathrm{~g}$ of smectite mixed or not with glutamic acid, serine, alanine and leucine to the basic mixture of smectite powder and water, either individually or collectively. Identical interacting sequences were made by adding the bacteria E. coli to the smectite powder in de-ionized water, either with or not amino acids and by shaking systematically for three weeks. The reference experiment corresponds to an interaction between the mineral and the solution without any bacteria or amino acid. After the three-week interaction, the clay material and the varied solutions were separated by centrifugation and the solutions analyzed for their element contents.

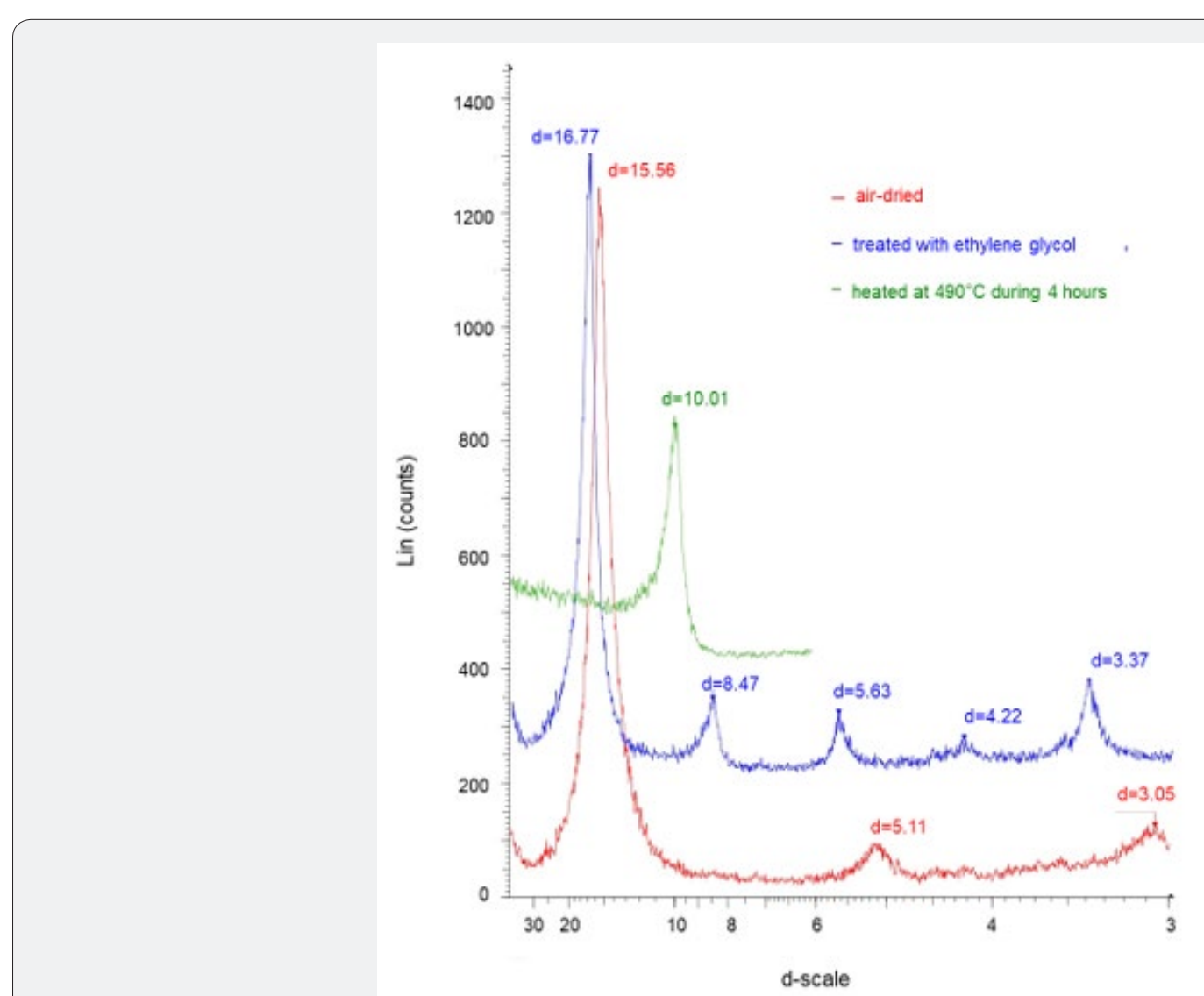

Figure 1: XRD patterns of the reference smectite mineral. The diagram of the untreated material is in red, that after ethylene-glycol treatment is in blue and that after heating is in green.

The major elements ( $\mathrm{Si}, \mathrm{Al}, \mathrm{Mg}, \mathrm{Ca}, \mathrm{Na}, \mathrm{K}$ and $\mathrm{P}$ ) and some trace elements $(\mathrm{Cu}, \mathrm{Zn}, \mathrm{Ba}, \mathrm{Fe}, \mathrm{Mn}$ and $\mathrm{Ag})$ were quantified by inductively coupled plasma atomic emission spectrometry (ICPAES) and inductively coupled plasma mass spectrometry (ICP-MS) was used to determine the amounts of the other trace elements ( $\mathrm{Cr}, \mathrm{Co}, \mathrm{Ni}, \mathrm{Rb}, \mathrm{Sr}, \mathrm{Zr}, \mathrm{U}$ and $\mathrm{Th}$ ) and the rare earth elements (REEs). A weekly routine analysis of the GL-O and BEN international mineral standards [18] provided an analytical precision of $\pm 2.5 \%$ for the major elements, $\pm 5 \%$ for the trace elements and $\pm 10 \%$ for the REEs, on the basis of the analytical procedure by Samuel et al. [19].

\section{Results}

To determine the impacts of the selected bacteria and amino acids on the clay material, the chemical composition of each solution was compared to that of the reference solution resulting from interaction of the mineral powder with pure de-ionized water. The hereunder database provides the $\mathrm{pH}$ and the elemental 
contents of the leachates in either micrograms or picograms per gram of smectite.

\section{The type of smectite}

The interacting experiments outline a systematic overwhelming release of $\mathrm{Na}$ in the leachates (Table 2). In turn, its amounts in the leachates represent up to about $90 \%$ of the leached major elements in most experiments, except for that involving $E$. coli either solely or mixed with serine and leucine. Such a large $\mathrm{Na}$ release points towards a Na-base smectite type [20] that yields generally a $\mathrm{Si} / \mathrm{Al}$ ratio of about 4.4 , which will be discussed in a further section.

\section{The pH values}

Table 1: $\mathrm{pH}$ values of the interacting fluids during the different experiments with the bacteria Escherichia and the amino acids.

\begin{tabular}{|c|c|c|}
\hline Composition of the Leachates & pH at Start of Experiment & pH at end of Experiment \\
\hline pure bi-distilled water & 9.12 & 10.3 \\
\hline smectite with water & 10.32 & 9.43 \\
\hline smectite with $E$. coli & 9.1 & 8.58 \\
\hline smectite with $E$. coli and glutamic acid & 4.2 & 9.2 \\
\hline Smectite with $E$. coli and alanine & 8 & 8.44 \\
\hline smectite with $E$. coli and serine & 6.7 & 9.66 \\
\hline smectite with $E$. coli and arginine & 10.2 & 9.02 \\
\hline smectite with $E$. coli, glutamic acid and arginine & 7.4 & 9.15 \\
\hline smectite with $E$. coli, arginine, serine and leucine & 8.5 & 8.29 \\
\hline smectite with $E$. coli, glutamic acid, serine and alanine & 6 & 8.28 \\
\hline smectite with $E$. coli, serine and leucine & 6.8 & 8.62 \\
\hline smectite with $E$. coli, glutamic acid, serine and leucine & 6.2 & 8.32 \\
\hline Smectite with glutamic acid & 4.3 & 10.7 \\
\hline smectite with arginine & 10.4 & 7.12 \\
\hline smectite with serine & 7.7 & 7.33 \\
\hline smectite with leucine & 8.1 & 7.53 \\
\hline smectite with alanine & 8.2 & \\
\hline
\end{tabular}

The $\mathrm{pH}$ of the de-ionized water used for the experiments was at 9.12 before any contact with the smectite or addition of either the bacteria or the amino acids (Table 1). This $\mathrm{pH}$ increased to 10.32 by adding the mineral powder, which suggests a discrete release of cations into the water, probably by desorption from crystal surfaces. Alternatively, addition of $E$. coli maintained the value of the $\mathrm{pH}$ close to that of the initial pure water at 9.10, not showing an immediate detectable interaction amongst the two components. In fact, this addition did not modify either the $\mathrm{pH}$ of the solution at the end of the experiment with only a slight increase from 9.10 to 9.43 . Addition of the amino acids to the smectite, water and E. coli mix decreased significantly most $\mathrm{pH}$ values, except that involving arginine, which gave a value close to the $\mathrm{pH}$ of the reference mixing of smectite and water. The most significant decrease was by addition of glutamic acid. During the experiments without E. coli, the pHs remained quite stable, except again when adding glutamic acid.

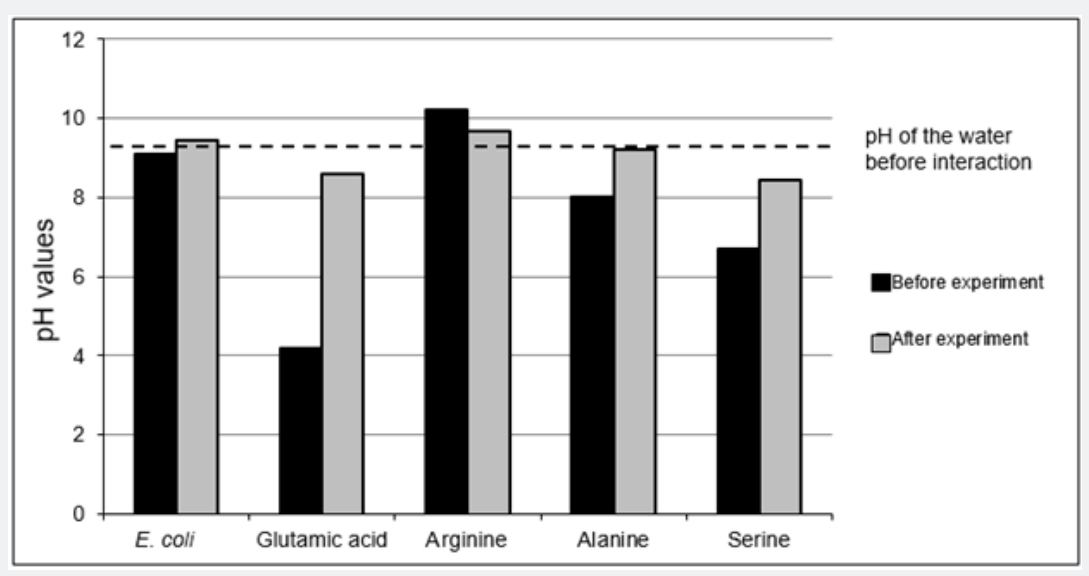

Figure 2: $\mathrm{pH}$ values of the solutions loaded either with $E$. coli or the amino acids at the start and the end of the experiments. 
Compared to the $\mathrm{pH}$ of 9.10 for the solution resulting from interaction between smectite and bacteria $E$. coli after three weeks of shaking, those of the solutions including the varied amino acids are significantly lower (Figure 2), except for the solution loaded with arginine. Compared to the initial $\mathrm{pH}$ (before interaction with amino acids), alanine, serine and glutamic acid combined with $E$. coli induced an increase of the $\mathrm{pH}$ from 8.00 to 9.20 , from 6.70 to 8.44 and from 4.20 to 8.58 , respectively. The last combination highlights indirectly a significant release of elements into the water, which almost doubles the value of the $\mathrm{pH}$.
The $\mathrm{pH}$ values of the batches with alanine and arginine are nearly similar after the three-week interaction to that of the leachate without amino acids. The pHs of the solutions with glutamic acid and with serine remained lower than that of the batch without amino acids (Figure 2). The ratio between the $\mathrm{pH}$ of each solution and that of the solution without any amino acid is less than unity for all solutions, except for that with arginine, which ratio is slightly above unity. In summary, the $\mathrm{pH}$ increases in the different solutions point, as expected, to exchange reactions between cations and $\mathrm{H}^{+}$and subsequent releases.

\section{The impacts of Escherichia coli on the smectite}

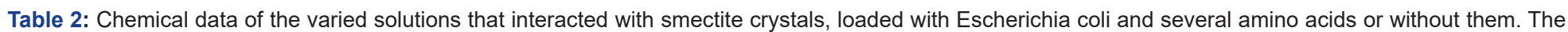
concentrations of major elements are expressed in $\mu \mathrm{g} / \mathrm{g}$ of the smectite, and those of the trace and REEs in pg/g.

\begin{tabular}{|c|c|c|c|c|c|c|c|c|c|c|c|c|c|c|}
\hline $\begin{array}{l}\text { Interaction of } \\
\text { Smectite }\end{array}$ & $\begin{array}{c}\mathrm{Si} \\
(\mu \mathrm{g} / \mathrm{g})\end{array}$ & $\begin{array}{c}\text { Al } \\
(\mu \mathrm{g} / \mathrm{g})\end{array}$ & $\begin{array}{c}M g \\
(\mu g / g)\end{array}$ & $\begin{array}{c}\mathrm{Ca} \\
(\mu \mathrm{g} / \mathrm{g})\end{array}$ & $\begin{array}{c}\text { Fe } \\
(\mu \mathrm{g} / \mathrm{g})\end{array}$ & $\begin{array}{c}\text { Mn } \\
(\mu \mathrm{g} / \mathrm{g})\end{array}$ & $\begin{array}{c}\mathrm{Ti} \\
(\mu \mathrm{g} / \mathrm{g})\end{array}$ & $\begin{array}{c}\mathrm{Na} \\
(\mu \mathrm{g} / \mathrm{g})\end{array}$ & $\begin{array}{c}K \\
(\mu g / g)\end{array}$ & Total & $\begin{array}{l}\mathrm{Na} / \\
\text { total }\end{array}$ & $\mathrm{Si} / \mathrm{Al}$ & $\mathrm{Ca} / \mathrm{Sr}$ & $\mathbf{K} / \mathbf{R b}$ \\
\hline with E. coli & 3536 & 784 & 200 & 97.8 & 820 & 2.72 & 28.7 & 12080 & 164 & 17713 & 0.68 & 4.51 & 66.1 & 449 \\
\hline $\begin{array}{l}\text { with } E \text {. coli and } \\
\text { glutamic acid }\end{array}$ & 432 & 0.32 & 129 & 504 & 0.6 & 0.32 & 0.08 & 17680 & 426 & 19172 & 0.92 & 1350 & 50.4 & 696 \\
\hline $\begin{array}{l}\text { with } E \text {. coli and } \\
\text { alanine }\end{array}$ & 488 & 15.4 & 15 & 76.4 & 14.7 & 0.56 & 0.72 & 16320 & 340 & 17271 & 0.94 & 31.7 & 40.6 & 991 \\
\hline $\begin{array}{c}\text { with } E \text {. coli and } \\
\text { serine }\end{array}$ & 384 & 0.08 & 282 & 2228 & 0.12 & 2.4 & bdl & 18000 & 459 & 21356 & 0.84 & 4800 & 66.6 & 450 \\
\hline $\begin{array}{l}\text { with } E \text {. coli and } \\
\text { arginine }\end{array}$ & 525 & 6.72 & 19.6 & 73 & 8.63 & 0.16 & 0.4 & 15280 & 253 & 16167 & 0.95 & 78.1 & 34.1 & 1552 \\
\hline $\begin{array}{c}\text { with } E \text {. coli, } \\
\text { glutamic acid and } \\
\text { arginine }\end{array}$ & 293 & 0.15 & 187 & 265 & 3.01 & 0.9 & bdl & 21450 & 785 & 22984 & 0.93 & 1953 & 11.8 & 384 \\
\hline $\begin{array}{l}\text { with } E \text {. coli, argi- } \\
\text { nine, serine and } \\
\text { leucine }\end{array}$ & 450 & 0.18 & 78.6 & 162 & 0.52 & 0.78 & 0.06 & 24900 & 1075 & 26667 & 0.93 & 2500 & 28.1 & 535 \\
\hline $\begin{array}{l}\text { with } E \text {. coli, glu- } \\
\text { tamic acid, serine } \\
\text { and alanine }\end{array}$ & 632 & 0.28 & 240 & 1612 & 0.47 & 1.6 & bdl & 13800 & 470 & 16756 & 0.82 & 2257 & 75.8 & 443 \\
\hline $\begin{array}{l}\text { with } E \text {. coli, serine, } \\
\text { and leucine }\end{array}$ & 435 & 0.06 & 327 & 2811 & 0.05 & 4.11 & bdl & 11280 & 309 & 15166 & 0.74 & 7250 & 79.9 & 379 \\
\hline $\begin{array}{l}\text { with E. coli, glu- } \\
\text { tamic acid, serine } \\
\text { and leucine }\end{array}$ & 426 & 0.39 & 51.9 & 441 & 0.48 & 0.24 & 0.03 & 9840 & 249 & 11009 & 0.89 & 1092 & 81.7 & 755 \\
\hline $\begin{array}{c}\text { reference leaching } \\
\text { with water }\end{array}$ & 12.3 & 0.46 & 3.81 & 8.2 & 0.2 & 0.04 & 0.004 & 272 & 3.04 & 300 & 0.91 & 26.7 & 56.6 & 426 \\
\hline $\begin{array}{l}\text { without } E \text {. coli with } \\
\text { glutamic acid }\end{array}$ & 476 & 1.88 & 440 & 1000 & 1.88 & 0.12 & 0.16 & 16360 & 368 & 18648 & 0.88 & 253 & 39.6 & 440 \\
\hline $\begin{array}{l}\text { without } E \text {. coli with } \\
\text { alanine }\end{array}$ & 568 & 34.5 & 86.4 & 310 & 28.5 & 1.36 & 1.4 & 14560 & 230 & 15820 & 0.92 & 16.5 & 11 & 849 \\
\hline $\begin{array}{l}\text { without } E \text {. coli with } \\
\text { serine }\end{array}$ & 816 & 0.12 & 738 & 828 & 0.34 & 0.42 & bdl & 25260 & 434 & 28077 & 0.9 & 6800 & 16.4 & 419 \\
\hline $\begin{array}{c}\text { without } E \text {. coli with } \\
\text { arginine }\end{array}$ & 474 & 2.45 & 22.8 & 208 & 1.68 & nd & 0.14 & 12312 & 142 & 13163 & 0.94 & 194 & 29.2 & 345 \\
\hline $\begin{array}{l}\text { without } E \text {. coli with } \\
\text { leucine }\end{array}$ & 760 & 82.2 & 97.6 & 351 & 70 & 1.68 & 3.64 & 13280 & 173 & 14819 & 0.9 & 9.25 & 56.6 & 420 \\
\hline
\end{tabular}

E stands for Escherichia, bdl for below detection limit and nd for not determined. 
International Journal of Environmental Sciences \& Natural Resources

\begin{tabular}{|c|c|c|c|c|c|c|c|c|}
\hline$=\frac{\frac{20}{200}}{30}$ & $\begin{array}{l}\infty \\
\stackrel{\infty}{\dot{m}}\end{array}$ & $\stackrel{\sim}{\sim}$ & $\stackrel{m}{\sim}$ & $\stackrel{\tilde{I}}{\exists}$ & N & $\underset{\infty}{\infty}$ & $\stackrel{\dot{q}}{\dot{q}}$ & : \\
\hline$\approx=\frac{\sqrt{20}}{00}$ & 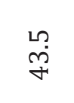 & $\stackrel{+}{\rightarrow}$ & 苞 & $\stackrel{N}{\stackrel{N}{i}}$ & $\begin{array}{l}\infty \\
\infty \\
\infty\end{array}$ & 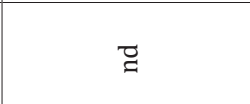 & $\stackrel{\vec{H}}{\stackrel{H}{二}}$ & $\stackrel{\infty}{\stackrel{\infty}{0}}$ \\
\hline $2 \frac{200}{200}$ & 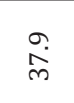 & $\begin{array}{l}\infty \\
\stackrel{\infty}{\infty} \\
\end{array}$ & $\underset{\sim}{\sim}$ & $\stackrel{+}{+}$ & $\stackrel{\sim}{\exists}$ & $\stackrel{n}{i}$ & $\stackrel{\text { 足 }}{\underset{\sim}{*}}$ & $\begin{array}{l}\Delta \\
\stackrel{\Delta}{ }\end{array}$ \\
\hline$\backsim \frac{\sqrt{00}}{30}$ & ô. & $\stackrel{m}{\sim}$ & $\underset{\sim}{\stackrel{H}{n}}$ & 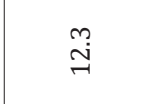 & $\stackrel{\text { }}{\stackrel{+}{+}}$ & $\begin{array}{c}m \\
\stackrel{m}{\not} \\
\stackrel{\rho}{+}\end{array}$ & $\begin{array}{l}\sigma \\
\stackrel{\sigma}{\dot{\rho}} \\
\dot{\beta}\end{array}$ & $\stackrel{\sim}{+}$ \\
\hline के & $\begin{array}{l}\dot{a} \\
\stackrel{-}{a}\end{array}$ & $\stackrel{+}{H}$ & $\stackrel{0}{\dot{m}}$ & $\stackrel{\grave{i}}{i}$ & $\stackrel{+}{+}$ & $\stackrel{\circ}{+}$ & 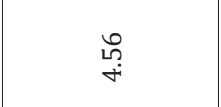 & $m$ \\
\hline$\approx \frac{\sqrt{00}}{30}$ & 品 & $\stackrel{N}{\exists}$ & $\stackrel{\leftrightarrow}{\mathrm{i}}$ & $\stackrel{\sim}{\sim}$ & $\begin{array}{l}0 \\
\vdots \\
0\end{array}$ & นึ้ & $m$ & ชి \\
\hline$\Xi \frac{\sqrt{00}}{00}$ & 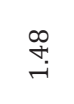 & 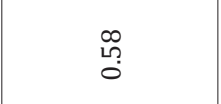 & 로 & $\stackrel{\infty}{\stackrel{\infty}{0}}$ & $\stackrel{\infty}{\infty}$ & fे & $\stackrel{\vec{r}}{+}$ & $\stackrel{\text { ्ְ? }}{\rightarrow}$ \\
\hline$\leqslant \frac{\frac{\sigma 0}{\partial 00}}{2}$ & $\underset{\substack{n \\
\rightarrow}}{\stackrel{n}{\sim}}$ & $\underset{\sim}{\stackrel{\sim}{*}}$ & $\stackrel{+}{\mathrm{N}}$ & $\stackrel{\sim}{\sim}$ & 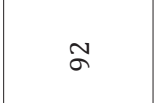 & $\begin{array}{l}\stackrel{L}{2} \\
\stackrel{\infty}{\longrightarrow}\end{array}$ & $\stackrel{\infty}{\dot{q}}$ & $\stackrel{+}{\stackrel{+}{\pi}}$ \\
\hline$>\frac{\sqrt{00}}{30}$ & $\stackrel{\text { Mn }}{\Omega}$ & 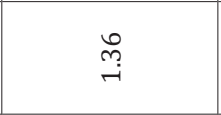 & $\begin{array}{l}\stackrel{m}{\alpha} \\
\dot{m}\end{array}$ & $\stackrel{2}{\stackrel{2}{0}}$ & $\stackrel{+}{\dot{H}}$ & $\stackrel{N}{\stackrel{N}{0}}$ & $\stackrel{\text { mे }}{\rightarrow}$ & $\underset{\infty}{\infty}$ \\
\hline 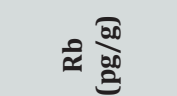 & $\begin{array}{l}\stackrel{L}{0} \\
\text { D }\end{array}$ & తี & $\stackrel{m}{m}$ & $\underset{\sim}{\stackrel{్}{\sim}}$ & శ్రి & $\stackrel{\text { L }}{\stackrel{\sim}{N}}$ & $\stackrel{\circ}{\stackrel{2}{N}}$ & $\underset{్}{\mathbb{U}}$ \\
\hline$<\frac{50}{a 00}$ & $\stackrel{\sim}{\sigma}$ & $F$ & $\begin{array}{l}\infty \\
\text { త్ }\end{array}$ & $\stackrel{p}{\text { r }}$ & $\stackrel{\infty}{\sim}$ & 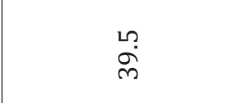 & $\stackrel{+}{\stackrel{N}{\Sigma}}$ & กี่ \\
\hline$z \frac{\sqrt{00}}{0.0}$ & 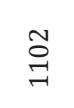 & $\underset{\sim}{\sim}$ & $\underset{\sim}{\stackrel{ి}{్}}$ & $\stackrel{\sim}{\sim}$ & $\underset{\sim}{+}$ & $\stackrel{\infty}{\stackrel{\infty}{\sim}}$ & $\stackrel{\stackrel{L}{m}}{\stackrel{m}{\sim}}$ & $\underset{\sim}{\stackrel{8}{2}}$ \\
\hline $8 \frac{\sqrt{00}}{30}$ & $\underset{\infty}{\vec{D}}$ & 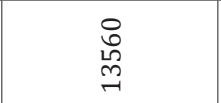 & $\stackrel{\circ}{\circ}$ & $\stackrel{\sim}{\stackrel{\sim}{\sim}}$ & $\underset{\sim}{\vec{b}}$ & $\stackrel{\text { N }}{\text {. }}$ & 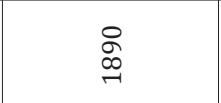 & ఖ \\
\hline$=\frac{\frac{00}{a 0}}{3}$ & 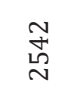 & 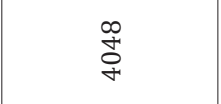 & $\stackrel{\infty}{\stackrel{\infty}{\sigma}}$ & 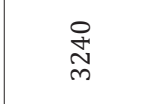 & 古 & $\stackrel{\circ}{\stackrel{\circ}{N}}$ & $\stackrel{N}{\widetilde{W}}$ & 号 \\
\hline$\Xi \frac{\sqrt[00]{00}}{3}$ & 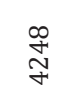 & 䀩 & $\underset{\sim}{\mathbb{N}}$ & $\stackrel{\infty}{\exists}$ & $\stackrel{+}{\Phi_{\infty}}$ & 号 & 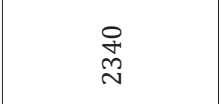 & $\underset{\sim}{\stackrel{N}{\sim}}$ \\
\hline$\Sigma \frac{\sqrt{00}}{200}$ & $\underset{\substack{m \\
=}}{=}$ & $N$ & $\ddot{\infty}$ & 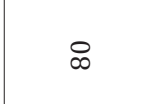 & స్ & $\stackrel{2}{\sigma}$ & ஃ & $N$ \\
\hline$>\frac{\frac{D 0}{00}}{3}$ & 亦 & $\stackrel{\text { N }}{\stackrel{N}{N}}$ & 号 & 辛 & $\underset{\sim}{\stackrel{\Xi}{*}}$ & 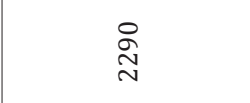 & ڤิ & 㞫 \\
\hline$\cong \frac{\sqrt{00}}{000}$ & $\stackrel{0}{0}$ & 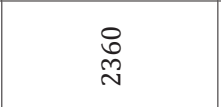 & $\underset{ }{\stackrel{8}{7}}$ & 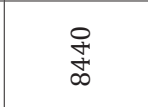 & $\stackrel{8}{8}$ & \&ి & $\begin{array}{l}\stackrel{8}{0} \\
\stackrel{2}{h}\end{array}$ & 勇 \\
\hline$\dot{\omega} \frac{\frac{\sigma 0}{\partial 0}}{20}$ & 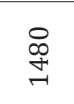 & @ & 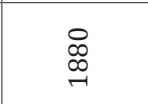 & 辛 & 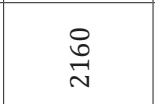 & 总 & $\begin{array}{l}8 \\
\text { in } \\
\text { in }\end{array}$ & $\begin{array}{l}\stackrel{\infty}{0} \\
\stackrel{\sim}{N}\end{array}$ \\
\hline $2 \frac{\sqrt{00}}{0}$ & min & $\begin{array}{l}\text { જે } \\
\text { }\end{array}$ & $\stackrel{m}{q}$ & ָे & 옷 & $\stackrel{\circ}{\stackrel{R}{R}}$ & $\exists$ & $\hat{\dot{q}}$ \\
\hline 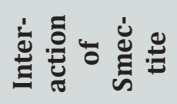 & 过: & 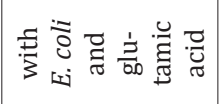 & 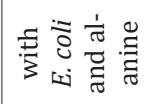 & 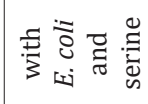 & 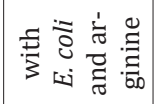 & 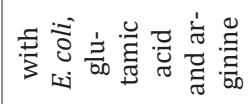 & 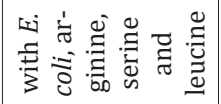 & 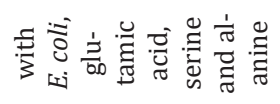 \\
\hline
\end{tabular}


International Journal of Environmental Sciences \& Natural Resources

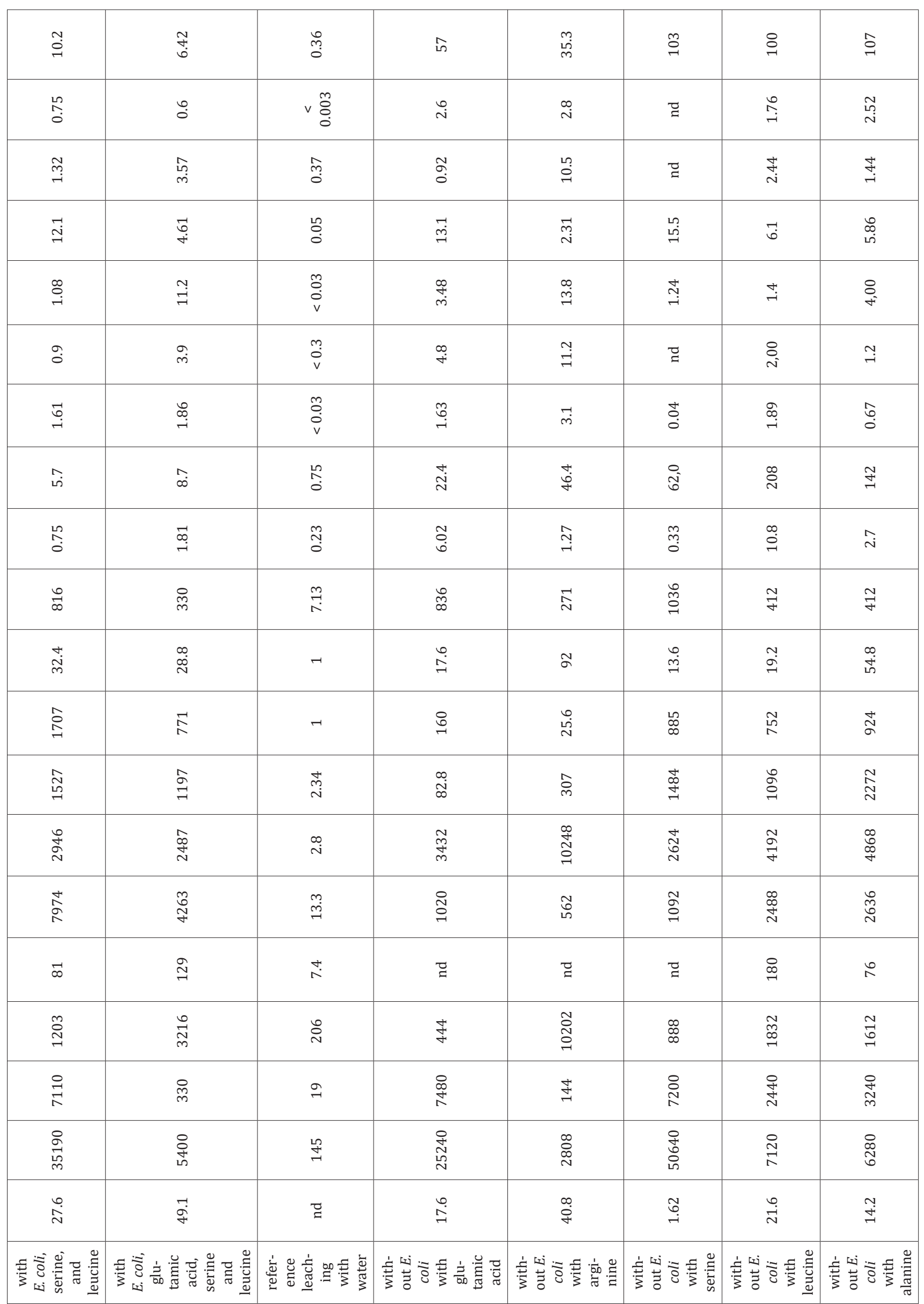




\begin{tabular}{|c|c|c|c|c|c|c|c|c|c|c|c|c|c|c|c|c|}
\hline 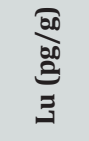 & $\stackrel{\widehat{\infty}}{\stackrel{\sim}{\sim}}$ & $\stackrel{0}{0}$ & oे & $\stackrel{\tilde{O}}{0}$ & $\bar{g}$ & $\stackrel{+}{0}$ & Õ. & $\hat{0}$ & $\tilde{O}$ & $\stackrel{+}{-}$ & $\begin{array}{l}\text { Oे } \\
0 \\
0 \\
\dot{v} \\
v\end{array}$ & 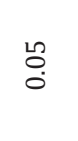 & $\bar{\Xi}$ & $\bar{g}$ & $\begin{array}{l}\stackrel{\infty}{\dddot{0}} \\
\stackrel{0}{0}\end{array}$ & oे \\
\hline 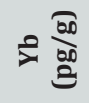 & $\stackrel{\infty}{\stackrel{\infty}{\sim}}$ & స̃ & ?ִ ? & J. & 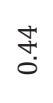 & $\stackrel{m}{0}$ & न्7 & 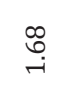 & $\stackrel{0}{\circ}$ & $\stackrel{\substack{0 \\
O}}{\circ}$ & 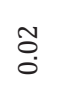 & స్ & $\stackrel{2}{\stackrel{2}{0}}$ & $\bar{g}$ & 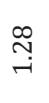 & $\stackrel{H}{\circ}$ \\
\hline$\xi \underbrace{\frac{\sqrt{D 0}}{\partial 0}}$ & 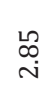 & 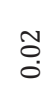 & $\begin{array}{l}\infty \\
0 \\
0\end{array}$ & $\bar{g}$ & $\bar{g}$ & $\bar{g}$ & $\bar{g}$ & $\hat{0}$ & $\bar{g}$ & $\stackrel{\circ}{\circ}$ & $\begin{array}{l}m \\
\stackrel{0}{0} \\
\dot{0} \\
\dot{v}\end{array}$ & $\stackrel{\text { L }}{0}$ & $\bar{\Xi}$ & $\bar{g}$ & $\stackrel{\infty}{0}$ & $\stackrel{\text { L }}{0}$ \\
\hline 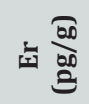 & $\stackrel{\text { i̊ }}{\text { in }}$ & $\stackrel{0}{0}$ & ㅊ. & $\stackrel{\circ}{0}$ & 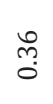 & $\begin{array}{l}\text { oे } \\
\dot{0}\end{array}$ & $\stackrel{m}{0}$ & $\stackrel{\mathbb{N}}{0}$ & $\stackrel{\circ}{\circ}$ & స̂. & $\stackrel{\tilde{O}}{0}$ & స్ & 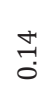 & $\bar{g}$ & 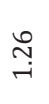 & 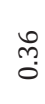 \\
\hline 舟 & $\stackrel{\stackrel{2}{ }}{\stackrel{2}{*}}$ & $\begin{array}{l}\text { ¿े } \\
\dot{0}\end{array}$ & $\stackrel{\text { N }}{0}$ & ठ̊. & $\bar{g}$ & $\bar{g}$ & $\begin{array}{l}\text { Dे } \\
\text { Oे }\end{array}$ & $\bar{\Xi}$ & $\stackrel{\overrightarrow{0}}{0}$ & $\stackrel{0}{\circ}$ & $\stackrel{0}{0}$ & $\begin{array}{l}0 \\
0 \\
0\end{array}$ & 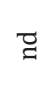 & $\bar{g}$ & $\stackrel{?}{0}$ & 궁 \\
\hline$\vec{a} \frac{\sqrt{\infty}}{0}$ & $\stackrel{\stackrel{\sim}{~}}{m}$ & ָָ & $\stackrel{\text { \&ै }}{\mathrm{r}}$ & $\stackrel{\infty}{0}$ & 丽 & ô & $\begin{array}{l}0 \\
\stackrel{0}{0}\end{array}$ & $\stackrel{L}{\stackrel{2}{0}}$ & $\stackrel{\text { L }}{\circ}$ & $\begin{array}{l}\vec{N} \\
\text { O }\end{array}$ & 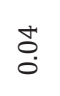 & $\stackrel{0}{\stackrel{0}{0}}$ & $\stackrel{\vec{H}}{\stackrel{\Delta}{0}}$ & $\bar{g}$ & $\stackrel{\stackrel{\sim}{N}}{\stackrel{N}{N}}$ & $\stackrel{\sharp}{\circ}$ \\
\hline$=\frac{b_{0}^{\infty 0}}{3}$ & नु & $\stackrel{m}{0}$ & $\stackrel{\text { ? }}{0}$ & $\bar{g}$ & $\bar{g}$ & $\widetilde{g}$ & 命 & $\bar{g}$ & $\bar{g}$ & $\begin{array}{l}m \\
\stackrel{0}{0}\end{array}$ & $\stackrel{\overrightarrow{0}}{0}$ & $\overrightarrow{0}$ & 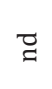 & $\bar{g}$ & mे & $\stackrel{\circ}{\circ}$ \\
\hline$=\underbrace{\frac{D}{\infty 00}}$ & ֻै & ֶֻ & $\stackrel{\text { fै }}{\mathrm{r}}$ & $\stackrel{\circ}{\circ}$ & $\stackrel{\widetilde{N}}{\tilde{0}}$ & 궁 & 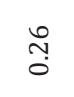 & $\begin{array}{l}\Delta \\
0 \\
0\end{array}$ & $\begin{array}{l}\text { ô } \\
\text { o. }\end{array}$ & $\overrightarrow{0}$ & 菅 & 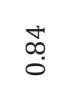 & $\stackrel{\vec{H}}{\circ}$ & $\begin{array}{l}\text { Oे } \\
\text { Oे }\end{array}$ & $\underset{N}{N}$ & 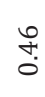 \\
\hline$=\frac{\sqrt{00}}{30}$ & $\stackrel{\infty}{=}$ & $\bar{g}$ & ָ̃ & $\bar{g}$ & $\bar{g}$ & $\bar{g}$ & $\bar{g}$ & $\bar{G}$ & $\bar{g}$ & $\stackrel{m}{\circ}$ & $\stackrel{\sigma}{0}$ & $\stackrel{\text { N }}{\text { Oे }}$ & $\bar{g}$ & $\bar{g}$ & $\begin{array}{l}\text { tे } \\
\text { d. }\end{array}$ & 궁 \\
\hline$E \frac{\infty}{\omega_{0}^{\infty}}$ & $\begin{array}{l}\infty \\
\substack{\infty \\
\infty}\end{array}$ & $\tilde{o}$ & ה. & 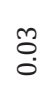 & $\tilde{N}$ & $\begin{array}{l}\infty \\
: \\
0\end{array}$ & $\stackrel{m}{0}$ & $\stackrel{\Delta}{0}$ & $\stackrel{L}{0}$ & $\stackrel{\circ}{\circ}$ & $\stackrel{\infty}{0}$ & th. & $\stackrel{0}{0}$ & $\bar{g}$ & $\overrightarrow{\vec{N}}$ & $\stackrel{\text { L }}{5}$ \\
\hline$=\frac{\sqrt{00}}{30}$ & $\underset{\sim}{\infty}$ & హ. & $\tilde{N}$ & ㄱ. & $N$ & $\stackrel{\text { L }}{0}$ & $\stackrel{\infty}{\stackrel{\infty}{0}}$ & $\stackrel{0}{0}$ & 궁 & $\stackrel{\text { N }}{\text { O }}$ & $\stackrel{m}{\stackrel{2}{0}}$ & $\stackrel{7}{\text { i }}$ & $\stackrel{H}{0}$ & $\bar{g}$ & 苛 & $\stackrel{\sim}{\stackrel{\sim}{\sim}}$ \\
\hline$=\frac{\sigma_{0}^{\infty}}{20}$ & $\stackrel{\mathscr{F}}{\dot{f}}$ & $\stackrel{\infty}{\stackrel{0}{0}}$ & $\stackrel{m}{+}$ & $\bar{g}$ & $\stackrel{+}{0}$ & $\begin{array}{l}\stackrel{0}{\circ} \\
\stackrel{0}{0}\end{array}$ & $\stackrel{\text { ने }}{\circ}$ & $\vec{g}$ & $\vec{g}$ & $\stackrel{+}{\circ}$ & $\stackrel{m}{0}$ & F & $\stackrel{0}{0}$ & $\bar{g}$ & $\overrightarrow{\mathrm{N}}$ & 苟 \\
\hline ن & 卢 & $\underset{\sim}{\mathbb{H}}$ & $\stackrel{\infty}{\dot{m}}$ & $\stackrel{\text { ֻొ }}{0}$ & $\stackrel{F}{+}$ & 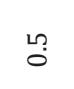 & $\stackrel{+}{\stackrel{\leftrightarrow}{\rightarrow}}$ & 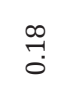 & $\stackrel{\infty}{\dddot{0}}$ & $\stackrel{\text { L }}{+}$ & $\stackrel{0}{\overrightarrow{0}}$ & $\tilde{N}$ & $\stackrel{\infty}{\circ}$ & 葛 & فे & $\begin{array}{l}\infty \\
\stackrel{\infty}{m} \\
\dot{m}\end{array}$ \\
\hline 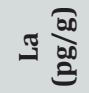 & $\underset{\sim}{\sigma}$ & 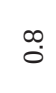 & $\underset{+}{+}$ & กี & $N$ & $\stackrel{m}{0}$ & $\stackrel{\infty}{\stackrel{\infty}{-}}$ & $\stackrel{\infty}{\stackrel{\infty}{0}}$ & 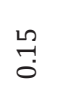 & $\stackrel{\vec{J}}{0}$ & $\overrightarrow{7}$ & $\stackrel{\text { ن }}{\text { ن }}$ & $\stackrel{+}{0}$ & $\stackrel{\leftrightarrow}{0}_{0}^{+}$ & $\begin{array}{l}\underbrace{+}_{\infty} \\
\infty\end{array}$ & $\stackrel{+}{\rightarrow}$ \\
\hline 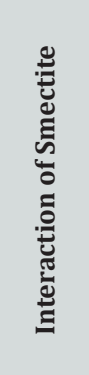 & 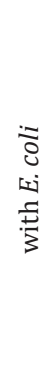 & 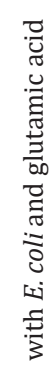 & 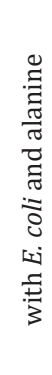 & 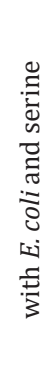 & 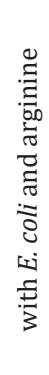 & 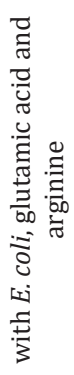 & 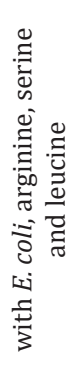 & 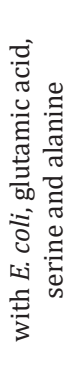 & 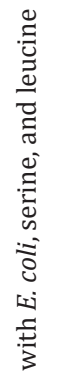 & 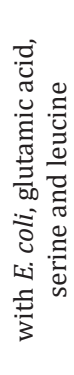 & 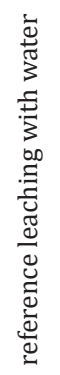 & 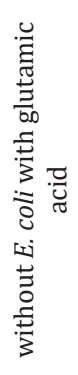 & 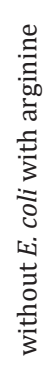 & 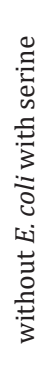 & 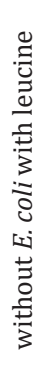 & 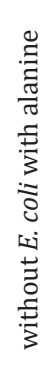 \\
\hline
\end{tabular}


The reference leaching of smectite with de-ionized water highlights only a significant removal of $\mathrm{Na}$, which confirms the already mentioned sodic nature of the selected mineral (Table 2 ), and that $\mathrm{Na}$ is easily removable from mineral structure. The contents of the other major elements are about 20 times less or even more than interaction with pure water, the leached Mn and Ti contents being the lowest. Most of the trace elements are in the range of $10 \mathrm{pg} / \mathrm{g}$ or even less, only Sr and V being in the 100 to $200 \mathrm{pg} / \mathrm{g}$ range and all REE contents are below $1 \mathrm{pg} / \mathrm{g}$. In summary, smectite leaching by pure water outlines a significant removal of $\mathrm{Na}$, Sr and V, while all other elements can be considered at the analytical uncertainty level, remaining in the mineral structure or at the crystal surfaces.

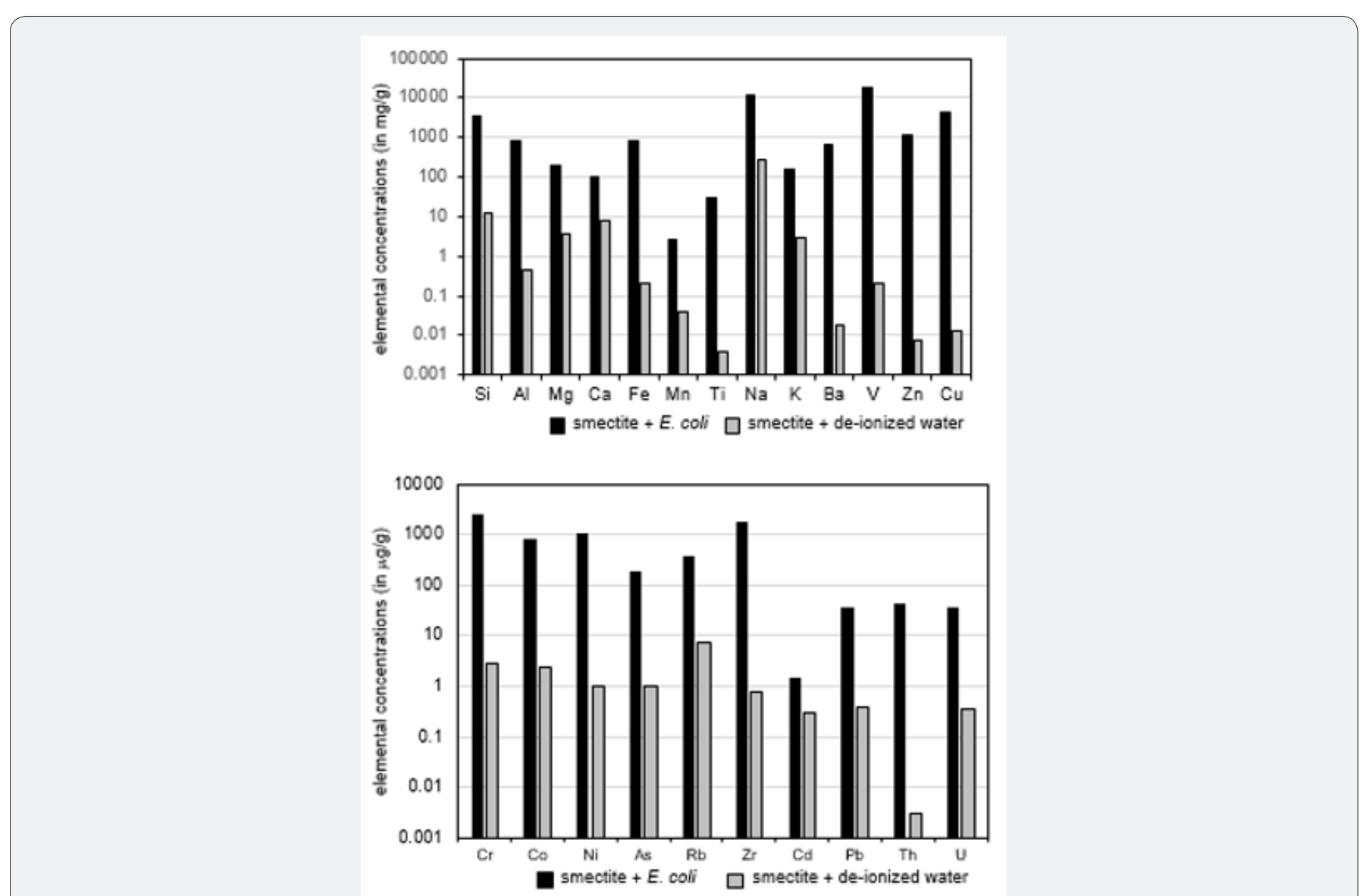

Figure 3: Leaching impact of E. coli on the major and trace elements from smectite compared to the leaching of the same elements by pure water.

The contents of elements leached from smectite with pure water were compared to those induced by interaction with $E$. coli (Table 2 \& Figure 3A). The bacteria induced larger removals of major elements than those obtained with pure water; the impact of $E$. coli is at a factor of about $50 \pm 10$ for $\mathrm{Na}, \mathrm{K}, \mathrm{Mn}$ and $\mathrm{Mg}$ relative to the pure water action. Leached $\mathrm{Ca}$ is 12 times lower, while those of $\mathrm{Si}(=287), \mathrm{Al}(=1704), \mathrm{Fe}(=4100)$ and $\mathrm{Ti}(=7175)$ are much higher. The Ti content could be due to the extremely low removal by the pure water, Ti being generally considered to be quite immobile in low-temperature surficial environments. The fact that the amounts of released $\mathrm{Na}, \mathrm{K}, \mathrm{Mn}$ and $\mathrm{Mg}$ are roughly similar suggests that they are most probably located in similar crystallographic sites of the interacting mineral. The low Ca removal is rather suggestive of the absence of Ca-carbonate(s) intergrown within the smectite crystals. The extremely high Fe release by the E. coli action suggests the occurrence of Fe- oxide crystals within the clay crystals that are preferentially dissolved by E. coli. The Si release could then relate to tetrahedral crystallographic sites that were "cleaned" by the bacteria together with some Al removal that could be indicative of tetrahedral $\mathrm{Al}$ leaching, an octahedral contribution being also possible.

The bacteria $E$. coli induced a leached $\mathrm{Si} / \mathrm{Al}$ ratio of 4.51 that is far from the same ratio obtained by either the amino acids or by combining the bacteria and amino acids. It is, therefore, tempting to attribute a mineral dissolution to $E$. coli rather than a desorption of components that are fundamental of the smectite structure. This possibility is indirectly corroborated by a low removal of $\mathrm{Na}$ by E. coli with a ratio of total removed $\mathrm{Na}$ / total removed elements much lower than those induced by the amino acids. In turn, the action of $E$. coli appears to rather remove elements from crystal structure than desorb elements from crystal surfaces. 
All trace elements are increasingly removed by the bacterial activity relative to the pure water leaching. The ratios among the amounts of $\mathrm{Ni}$ and $\mathrm{Zr}$ released by the bacteria relative to those removed by the plain water are significantly beyond 1000, those of $\mathrm{Zn}, \mathrm{Cu}, \mathrm{Cr}, \mathrm{Co}, \mathrm{As}, \mathrm{Y}, \mathrm{Cs}, \mathrm{Pb}$ and $\mathrm{U}$ being around 100 , and those of $\mathrm{Ba}, \mathrm{V}$ and $\mathrm{Rb}$ below 100 (Table 2 \& Figure 3B). An easy way to set relative variations of the REEs is a comparison with a reference [21]. Here, we have chosen the amounts of REEs leached by pure water as the reference. Relative to released REEs by pure-water leaching, those removed by the bacteria exhibit a significant fractionation with a decrease in light REEs (LREEs) from La to $\mathrm{Tb}$ including a slight positive Ce and a negative Eu anomaly that, however, are not analytically significant (Figure 4A). The decrease is fairly linear from La at a ratio of about 1700 to $\mathrm{Tb}$ at a ratio of about 800 , continuing with a flat distribution pattern towards Lu.

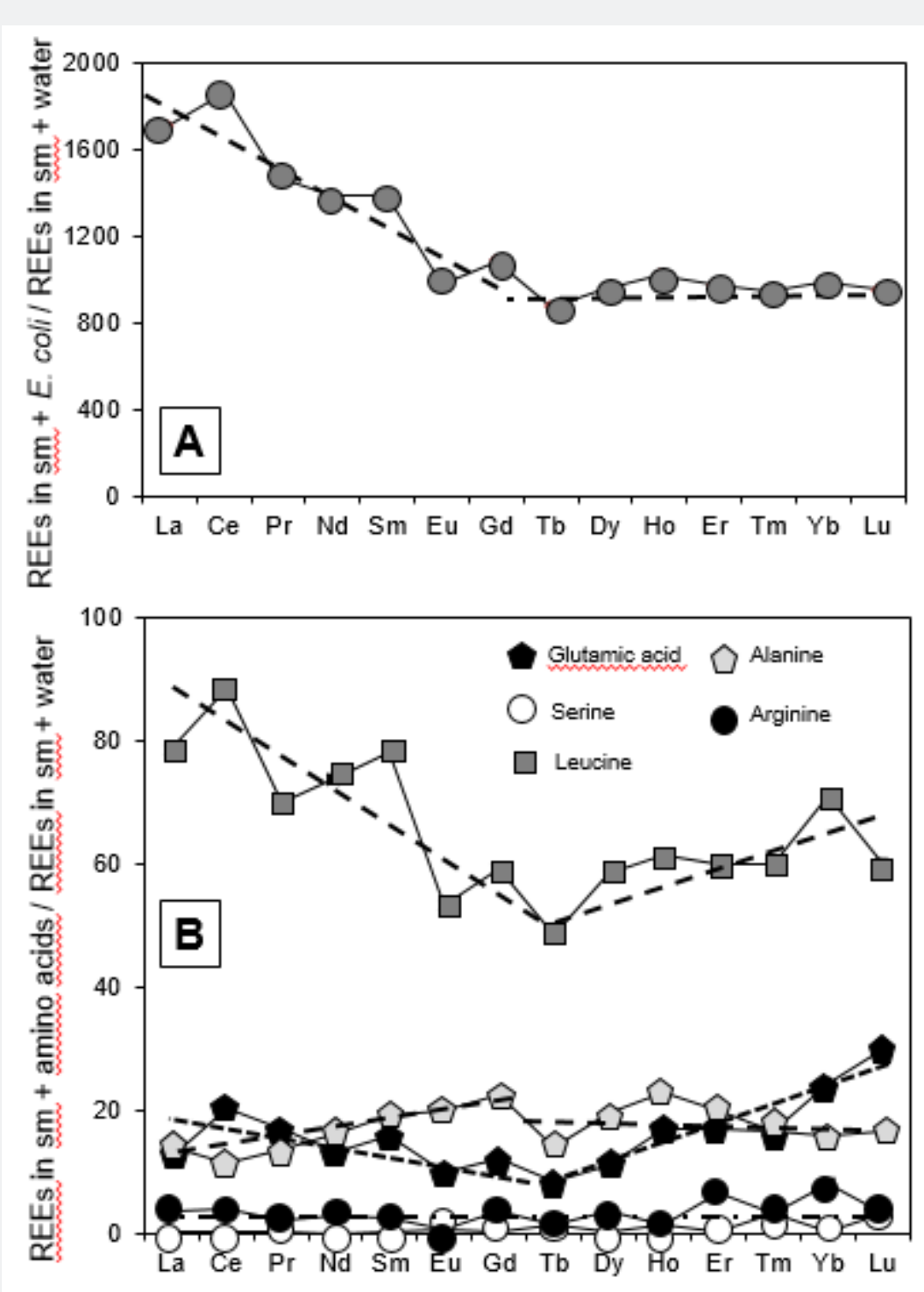

Figure 4: Distribution patterns of the REEs removed by $E$. coli and by the different amino acids relative to their distribution in the leachates by pure water.

\section{The action of the amino acids}

Expectedly, all selected amino acids induce a more pronounced leaching of major, trace and REEs from smectite crystals than pure water (Table 2). Again the highest efficiency of the amino acids is sketched by the amount of removed Na. Concerning the major elements, the occurrence of serine appears to be the most efficient with a maximum removal of $\mathrm{Si}, \mathrm{Mg}$, $\mathrm{Na}$ and $\mathrm{K}$, and the less efficient for the releases of $\mathrm{Al}$ and $\mathrm{Fe}$. The addition of leucine to the water removes mostly $\mathrm{Al}, \mathrm{Fe}, \mathrm{Mn}$ and $\mathrm{Ti}$. Addition of alanine to water is never the most efficient for any major element, but it is the second most efficient to remove $\mathrm{Al}, \mathrm{Mg}, \mathrm{Fe}, \mathrm{Mn}$ and Ti. This is also the case for arginine, which is a second-order remover of $\mathrm{Si}, \mathrm{Mg}$, $\mathrm{Na}$ and $\mathrm{K}$. 
Addition of glutamic acid to the water makes it the best remover of $\mathrm{Ca}$ and the second-best remover of $\mathrm{Na}$ and $\mathrm{K}$.

The relative efficiency of the amino acids has been evaluated on Si by comparing its leached amounts by the amino acids to that removed by pure water (Figure 5). For most amino acids, the ratio of released $\mathrm{Si}$ is beyond unity that is to say that the amino acids removed more Si than pure water. In fact, only arginine removed significantly more than water only, at a ratio of about 3 , whereas glutamic acid, leucine and alanine removed less at a ratio between 1.2 to 1.7 , serine removing even less than water at a ratio of 0.2 .

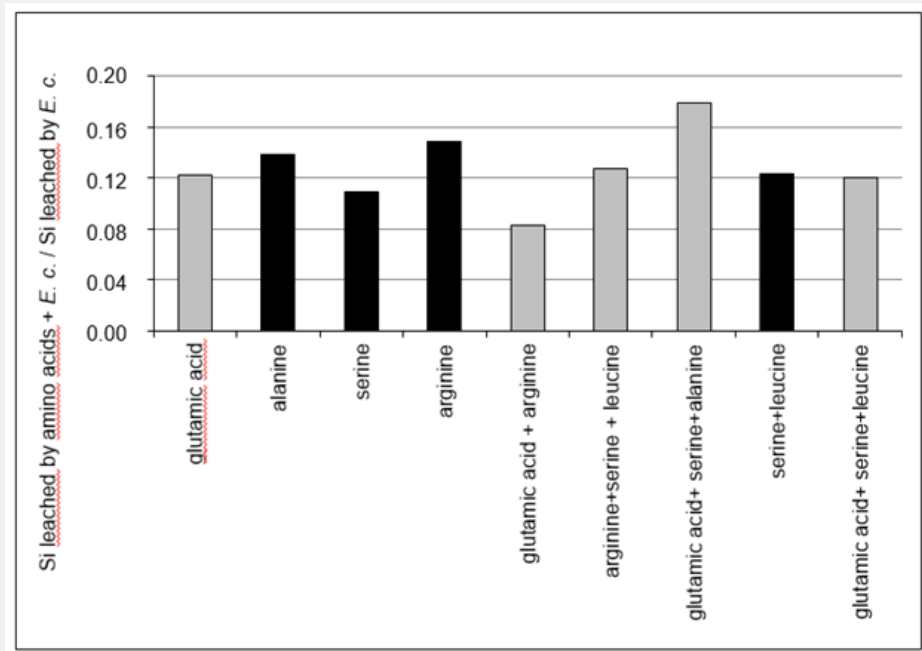

Figure 5: Amounts of $\mathrm{Si}$ leached by mixtures of amino acids and $E$. coli relative to the amounts of Si leached by $E$. coli only. In black are the amounts induced by the different amino acids and in gray the amounts released by the glutamic acid combined in different ways.

In summary, the most released amounts of $\mathrm{Si}, \mathrm{Al}, \mathrm{Mn}$ and $\mathrm{Fe}$ from smectite characterize the batch without amino acids, while the most released $\mathrm{Ca}$ and $\mathrm{K}$ were by amino acids. Removed $\mathrm{Mg}$ increased only when serine was added to the solution. Relative to the reference water data, serine induced higher leaching of $\mathrm{Ca}, \mathrm{K}$ and $\mathrm{Mg}$ than the other amino acids. Conversely, addition of serine reduced more the leaching of $\mathrm{Al}, \mathrm{Si}$ and Fe than with the other amino acids, all being known to activate bacterial life in soils $[22,23]$. Indeed, previous studies reported the inhibition of bacteria growth by amino acids that can also modify the bacterial phenotypes, some amino acids being even toxic to bacteria $[24,25]$. The amounts of leached $\mathrm{K}, \mathrm{Al}$ and $\mathrm{Na}$ by amino acids are higher than without any of them. Such a difference suggests an induced desorption process with the potential crystallization of organic complexes.

Relative to the elements released into the solution without amino acids, the solutions with amino acids induced depleted releases of Cd, As, Pb, Th, Zn and U (Figure 3B). Elements such as $\mathrm{Cr}, \mathrm{Ni}$ and $\mathrm{Co}$ increased by interaction with solutions containing glutamic acid and alanine and decreased by interacting with arginine and serine solutions. Barium was enriched in all solutions with amino acids with even a higher leaching in the presence of serine. Rubidium increased in the solutions with glutamic acid and serine, while $\mathrm{Cu}$ increased only by interacting with the solution containing glutamic acid.

Addition of amino acids increased the sorption ability of the light REEs (LREEs from La to Eu) compared to the heavy
REEs (HREEs). Koeppenkastrop et al. [26] described a similar fractionation trend to that found here, i.e., preference for sorption of LREEs, which can result from greater tendency of HREEs to integrate carbonate complexes compared to the preferentially adsorbed LREEs. In turn, amino acids seem to influence the REE behavior due to the fact that organic complexes may influence the sorption of metallic elements at the surface of oxides [27]. In the detail, the distribution spectra of the leached REEs can, again, be compared to that induced by pure water (Figure 4). Some patterns are completely flat from $\mathrm{La}$ to $\mathrm{Lu}$, which is the case for those recovered from serine and arginine action, giving low ratios relative to the action of pure water. The whole REE pattern by alanine interaction is also quite flat. However, it is more scattered than the two previous ones, with a ratio relative to the action of pure water at about 20 . The two other patterns resulting from interaction with glutamic acid and leucine are somewhat similar with a decrease of the ratios from La to $\mathrm{Tb}$ and an increase for the HREEs until Lu. Interestingly, the two REE distribution patterns are quite similar along a downward trend followed by an upward one.

\section{The combined action of Escherichia coli and the amino acids}

The three-week interaction of $E$. coli with smectite released $53 \mathrm{pg} / \mathrm{g}$ of $\mathrm{P}$, whereas addition of either glutamic acid or arginine induced a higher $\mathrm{P}$ release. This $\mathrm{P}$ release increased with the $\mathrm{pH}$, as for $\mathrm{Al}$ (correlation coefficient $\mathrm{r}^{2}=0.6$ for both elements), which suggests a combined process of dissolution/desorption related to 
a process involving complexes of organics. Hence, Huang \& Keller [28] reported that organic acids enhance the extraction of Si and Al from clay crystals, which cannot be excluded here with much higher releases of Si than of Al. Conversely, the enhanced effects of amino acids are controlled by concentrations of amino-acid cationic species that interact with the negatively charged $\mathrm{SiO}^{-}$ sites at the surface of amorphous silica, but also of phyllosilicates, as observed here. Lovering [29] reported also notably high concentrations of dissolved $\mathrm{Si}$ and $\mathrm{Al}$ in association with organic substances together with the accumulation of given elements and removal of others, due to chelation or complexation.

The combined action of $E$. coli together with amino acids has also been examined. In the case of the Si leaching for instance, most selected amino acids added to E. coli decrease significantly the impact of the bacteria to about $12 \%$, which represents another argument for differentiated actions by E. coli and the amino acids (Figure 5). For instance, alanine, serine and arginine added individually to $E$. coli reduce the leaching of Si to similar amounts. In the case of a combined addition of glutamic acid and arginine, the release of Si decreased also, by a similar amount of 12 to $18 \%$. This amount has also been obtained by combining glutamic acid, serine and leucine with E. coli. In summary, addition of amino acids to E. coli systematically decreases the leaching efficiency of E. coli by as much as about 80 to $90 \%$.

Interestingly, leaching of smectite with pure water released here more HREEs than in the presence of $E$. coli. The solution with amino acids also released more HREEs (Figure 4), for instance with alanine. The pattern of the extracted REEs seems to result mainly from a desorption process off the clay crystals.

\section{The combined action of the amino acids}

Release of $\mathrm{K}, \mathrm{P}$ and $\mathrm{Na}$ is significant here with a combined addition of arginine, serine and leucine to the interacting solution. Silicium, Mg and Mn are also more removed from smectite when glutamic acid is combined with serine and alanine. Comparison between the reaction of the clay material with individual and several amino acids indicates that glutamic acid together with arginine reduced significantly the releases of $\mathrm{Si}, \mathrm{Al}, \mathrm{Fe}$ and $\mathrm{Mn}$, but increased those of $\mathrm{Mg}, \mathrm{K}, \mathrm{Na}$ and $\mathrm{Ca}$. More $\mathrm{P}$ was released from solution with glutamic acid combined with arginine than by reacting only with glutamic acid, but similarly when reacting with serine only.

The releases of $\mathrm{Si}, \mathrm{Al}$ and $\mathrm{P}$ during interaction of the smectite with glutamic acid, serine and alanine are lower than by interaction with only glutamic acid, but higher than with serine only. To the opposite, the releases of $\mathrm{Mg}$ and $\mathrm{Ca}$ are higher in a mixture of glutamic acid, serine and alanine than with glutamic acid, but lower than with serine only. Iron release is much less when reacting with a mixture consisting of glutamic acid, serine and alanine compared to the impact of the sole glutamic acid, but it is more released than with the serine solution. Potassium is more released by a mixture of glutamic acid, serine and alanine, than by a solution containing only serine or glutamic acid. The action of combined amino acids is not the same for all trace elements. For instance, $\mathrm{Sr}$ is more released when serine is combined with leucine, whereas $\mathrm{Ba}$ is more dissolved in a solution with glutamic acid, serine and alanine, Co in a solution with arginine, serine and leucine, Ni in a solution with glutamic acid, serine and alanine, and $\mathrm{Rb}$ in a solution with glutamic acid and arginine.

The enrichment in leached HREEs is less significant with a solution containing a mixture of glutamic acid and arginine than with a solution containing only arginine. The association of serine with leucine or of leucine with glutamic acid buffers the leaching of the LREEs, which is visible when the reaction involves only serine. When only alanine is involved, the ratio $\mathrm{La} / \mathrm{Yb}$ changes significantly from 0.15 to about 0.01 for a combined action by glutamic acid and serine.

\section{Discussion}

A ratio between elements removed in the absence of $E$. coli and/or amino acids and the same elements released in the presence of the bacteria and/or the amino acids has been calculated to evaluate the dissolution/desorption releases specific to each used reactant. The ratios above unity identify increased removals, while ratios below unity indicate inhibited dissolution or dominant adsorption.

\section{Dissolution rates of the major elements}

Released materials from smectite stimulated by E. coli indicate that about $12 \mu \mathrm{g} / \mathrm{g}$ of Na were removed from mineral after a threeweek interaction. The second highest removed amount is for $\mathrm{Si}$ with $3.5 \mu \mathrm{g} / \mathrm{g}$, all other released amounts being below $1 \mu \mathrm{g} / \mathrm{g}$ : $0.82 \mu \mathrm{g} / \mathrm{g} \mathrm{Fe}, 0.78 \mu \mathrm{g} / \mathrm{g} \mathrm{Al}, 0.53 \mu \mathrm{g} / \mathrm{g} \mathrm{P}, 0.20 \mu \mathrm{g} / \mathrm{g} \mathrm{Mg}, 0.16 \mu \mathrm{g} / \mathrm{g} \mathrm{K}$, $0.10 \mu \mathrm{g} / \mathrm{g} \mathrm{Ca}, 0.03 \mu \mathrm{g} / \mathrm{g} \mathrm{Ti}$, and $0.003 \mu \mathrm{g} / \mathrm{g} \mathrm{Mn}$. Addition of amino acids to the solution increased the release rates of $\mathrm{Na}$ and $\mathrm{K}$ and decreased those of $\mathrm{Si}, \mathrm{Al}, \mathrm{Ti}, \mathrm{Mn}$ and $\mathrm{Fe}$. The release rate of $\mathrm{Ca}$ increased with the solution containing glutamic acid and serine, while that of $\mathrm{Mg}$ increased with the solution containing serine, and that of $\mathrm{P}$ with the solution containing glutamic acid and arginine.

In fact, addition of amino acids can modify the number of microorganisms in solution and, therefore, can decrease the activity of E. coli in the water-solid mixture, unless it is due to increased acidity. Cations such as $\mathrm{Si}, \mathrm{Al}$ and $\mathrm{Fe}$ are found to be less soluble with complexing organic acids than in pure water, which is to the opposite to $\mathrm{Na}, \mathrm{Ca}, \mathrm{K}$ and $\mathrm{Mg}$ that are found to be more soluble in solutions with amino acids. Dissolution of Fe parallels that of $\mathrm{Al}$ (Figure 3) and the relatively depleted removal of $\mathrm{Al}$ and Fe by organic acids might then be due to a chelation process by the acids followed by an adsorption at the surface of the mineral crystals. 
The increase of $\mathrm{Ca}, \mathrm{Na}$, and $\mathrm{K}$ in the leachates cannot be due to selective removals by amino acids, since all their contents increase, which has already been reported by Huang \& Keller [28]. Dissolution of $\mathrm{Ca}$, Na and $\mathrm{K}$ should, therefore, be considered apart from that of the other elements for several reasons. They may be associated with the smectite-type clay or be simply adsorbed at the crystal surface as oxides, being removed by ion-exchange reactions rather than by dissolution of the mineral structure. None of these elements is among the constitutive elements of the selected clay material. Furthermore, they are typically highly soluble in pure water so that chelation processes may not be detectable. Calcium, Na and $\mathrm{K}$ may then be released from clay materials by ion-exchange reactions. One specific result is that $\mathrm{Ca}$ and $\mathrm{Na}$ are strongly and preferentially dissolved by serine compared to the other amino acids.

\section{Meaning of the released $\mathrm{Si} / \mathrm{Al}$ ratio}

The amounts of leached Si and $\mathrm{Al}$, and possibly REEs, probably identify partial dissolution of and adsorption on the crystal structures for the two formers and cation desorption for the REEs. This is confirmed here by a significantly higher release of Al from smectite in presence of $E$. coli than in its absence (Figure 3). Similar results were observed for $\mathrm{Si}$ with a generated $\mathrm{Si} / \mathrm{Al}$ ratio of about 4.5 and 26.7 in the presence and absence of E. coli, respectively. Interestingly, the leached $\mathrm{Si} / \mathrm{Al}$ ratio of the Na-smectite is also at 4.5 by interaction with pure water. Alternatively, addition of amino acids reduced significantly the amount of released $\mathrm{Al}$, the most important decrease being observed by a combined action of serine and leucine. This decrease confirms the impact of amino acids on the activity of microorganisms such as E. coli, as well as their function as crystal surface cleaners rather than crystal assemblage dissolvers.

The concentrations of $\mathrm{Si}$ and $\mathrm{Al}$ in the dissolution solutions have already been used for calculation of reaction rates [30$32]$, and for evaluation of the stoichiometry of alumino-silicate dissolutions [33]. The differences between the stoichiometry of $\mathrm{Si}$ and $\mathrm{Al}$ released during mineral dissolution or leaching of adsorbed elements are consequences of the activity at the crystalsolution interface. The processes include the actual dissolution of the mineral and the subsequent ion sorption or exchange at the mineral surfaces [34]. Zyssed \& Schindler [30] showed that the $\mathrm{Si}$ and $\mathrm{Al}$ dissolution rates of a K-montmorillonite decrease with increasing $\mathrm{pH}$, which is the case here for a similar Na-smectite. Changes of $\mathrm{pH}$ are caused by protonation of the surface edges of the clay crystals and the ion exchange reaction between hydrogen and the exchangeable cations, as well as here by the addition of amino acids that reduces the activity of the associated $E$. coli.

To illustrate the difference between the releases of $\mathrm{Si}$ and $\mathrm{Al}$, Sondia et al. [35] considered that the ratio $(\mathrm{Al} / \mathrm{Si})_{\text {aq }} /(\mathrm{Al} / \mathrm{Si})_{\text {solid }}<1$ indicates a preferential retention of $\mathrm{Al}$ by the mineral relative to $\mathrm{Si}$, while a $(\mathrm{Al} / \mathrm{Si})_{\mathrm{aq}} /(\mathrm{Al} / \mathrm{Si})_{\text {solid }}>1$ indicates a higher release of $\mathrm{Al}$. On the basis of an average composition of smectite from a bentonite unit obtained by Karnland [36] and Abdullahi \& Audu [37], the $(\mathrm{Al} / \mathrm{Si})_{\mathrm{aq}} /(\mathrm{Al} / \mathrm{Si})_{\text {solid }}$ is lower than 1 for all solutions, which indicates that $\mathrm{Si}$ is more released than $\mathrm{Al}$. However, it cannot be ignored that quartz grains could have been naturally mixed with the bentonite material.

\section{About the $\mathrm{K} / \mathrm{Rb}, \mathrm{Ca} / \mathrm{Sr}$ and $\mathrm{U} / \mathrm{Th}$ ratios}

It is commonly admitted that $\mathrm{Ca}$ and $\mathrm{Sr}$ are chemical elements that have similar behaviors in Earth near-surface processes $[38,39]$, the main difference being in their relative contents. It is, therefore, of interest to follow how their ratio changes depending on the interactions between mineral and E. coli, as well as between the same mineral and different amino acids. For the same reasons, an evaluation of the $\mathrm{K} / \mathrm{Rb}$ and $\mathrm{U} / \mathrm{Th}$ ratios might also be instructive in the same interactive system.

Here, the interaction of smectite with water containing E. coli does not reflect a significant change in the dissolution selectivity of $K$ relative to $R b$, since the $K / R b$ ratio for dissolved smectite in pure water at 426 is nearly the same in presence of $E$. coli at 450. This inclines one to consider that the two elements are probably tied to the same crystallographic sites of the mineral. Conversely to $\mathrm{K}$ and $\mathrm{Rb}, E$ coli induced a selective dissolution of $\mathrm{U}$ relative to $\mathrm{Th}$, and Ca relative to $\mathrm{Sr}$, compared to the dissolution rates in the absence of $E$. coli. The relative increase in the $\mathrm{K} / \mathrm{Rb}$ ratio of the leachates with glutamic acid, alanine and arginine points to a selective dissolution of $\mathrm{K}$ relative to $\mathrm{Rb}$ (Figure 6). Only serine induced a $\mathrm{K} / \mathrm{Rb}$ ratio similar to that of the solution without any amino acid $(\mathrm{K} / \mathrm{Rb}$ at 450$)$, relative to the ratios resulting from action of the other amino acids. The varied increases suggest that there is a higher organic contribution in the $\mathrm{K} / \mathrm{Rb}$ ratio of the leachates depending on the type of amino acid. Chaudhuri et al. [40] reported ratios of 50 to 650 for silicate minerals and higher ranges, from 165 to 47000 , for organic materials. Characterized by $\mathrm{K} / \mathrm{Rb}$ ratios from 991 to 1552, the solutions obtained from arginine and alanine interactions could, then, reflect an organic contribution, which suggests in turn that these two amino acids preferentially remove $\mathrm{K}$ from organic compounds that were probably present in non-detectable small amounts in the initial mineral separate.

As for the $\mathrm{K} / \mathrm{Rb}$ ratio, the $\mathrm{Ca} / \mathrm{Sr}$ ratio observed in the solution with serine is similar to that in the solution without any amino acid, which implies that serine has no specific impact on these elements. The increase of Sr and the decrease of Ca in the solutions with glutamic acid, arginine and alanine induced a decrease in the $\mathrm{Ca} / \mathrm{Sr}$ ratio that is more significant than for interaction with serine and glutamic acid in which both the $\mathrm{Sr}$ and $\mathrm{Ca}$ increase relative to the interaction with pure water without amino acids. Furthermore, the limited variation of $\mathrm{Ca} / \mathrm{Sr}$ in these solutions indicates that there is no selective release of $\mathrm{Sr}$ and $\mathrm{Ca}$, which is to the opposite of interactions with solutions with alanine and arginine. 
In summary, arginine is the only amino acid that induces a differential extraction of $\mathrm{K}$ and $\mathrm{Rb}$ and of $\mathrm{Ca}$ and $\mathrm{Sr}$ during the interaction: the ratios are highest for $\mathrm{K} / \mathrm{Rb}$ and lowest for $\mathrm{Ca} /$ $\mathrm{Sr}$, suggesting again the occurrence of a few organic compounds hidden in the initial smectite fraction. In the case of the U/Th ratio at 0.82 in the pure water solution, it increased slightly in all solutions with either amino acids or E. coli and significantly in the case of alanine, which suggests some discrete fractionation during the alteration of bentonite may be, again, between the organic and inorganic components (Figure 6).
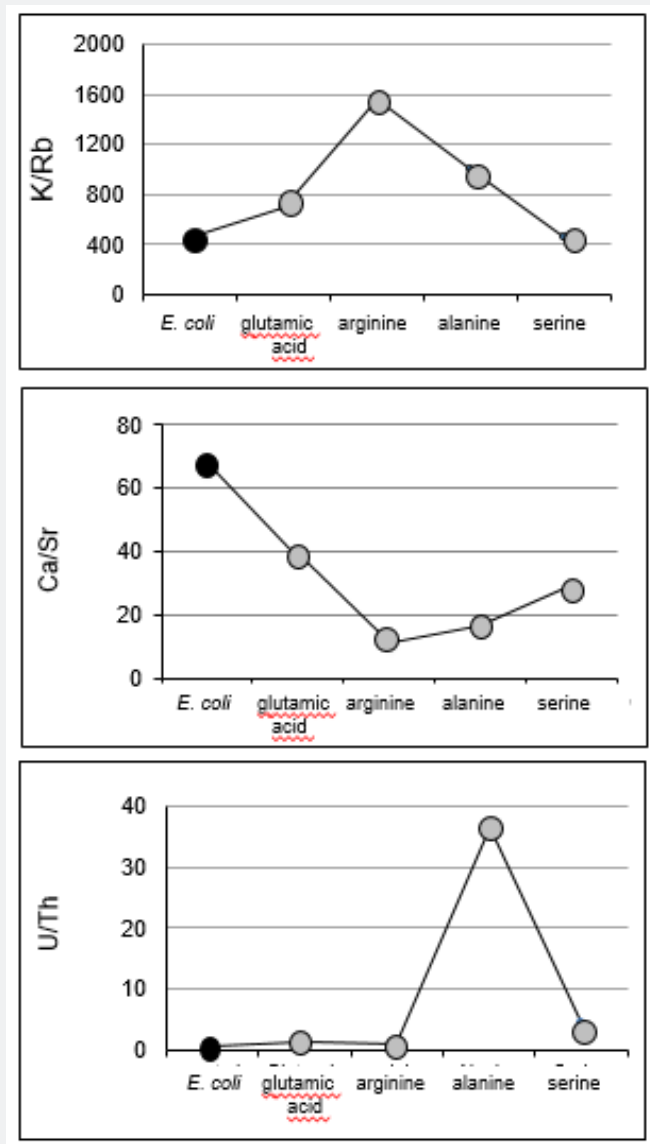

Figure 6: Released K/Rb, Ca/Sr and U/Th ratios by $\mathrm{E}$. coli (as a black dot) and by different amino acids (as gray dots).

\section{The impact of Escherichia coli relative to that of the amino acids}

The global action of E. coli compared to that of the amino acids used here outlines a high removal of Si and Fe relative to negligibly low removals by any amino acid (Figure 3). Conversely, the impact of $E$. coli is negligible in the case of $\mathrm{Ca}$, while serine is the most efficient. Interestingly, addition of $E$. coli to the leaching water is not as efficient for $\mathrm{Na}$ as it is for any of the amino acids, while its action on Mg is again highest with serine. Removal of the trace elements highlights a predominant leaching of As and $\mathrm{Zn}$ by E. coli (Figure 3B), glutamic acid being the most efficient remover of $\mathrm{Cr}$, $\mathrm{Co}, \mathrm{Ni}$ and $\mathrm{Cu}$. Addition of serine is most efficient for removal of $\mathrm{Rb}$, while alanine released more $\mathrm{Cr}$ than the other amino acids.

The selected smectite consists of a tetrahedral-octahedraltetrahedral (T-O-T) framework, like illite for instance or micas. Its tetrahedral sites are mainly occupied by $\mathrm{Si}$ and less by $\mathrm{Al}$, and the octahedral sites are mostly occupied by the leftover Al, Fe and/ or $\mathrm{Mg}$, depending on the origin of the mineral. The interlayers between the octahedral layers are occupied partially by $\mathrm{K}[41,42]$ that is not tied to this "flat" organization. As E. coli mostly removed $\mathrm{Si}$ and Al during the present experiments, it can be considered that it dismantled partly the basic T-O-T organization by removing $\mathrm{Si}$ and $\mathrm{Al}$ of accessible tetrahedral and/or octahedral sites. On the other hand, as $\mathrm{Na}, \mathrm{Ca}, \mathrm{K}$ and the trace elements are not part of the mineral framework, they were most probably adsorbed at the surface of the crystals or represent soluble minerals that were successfully dissolved and leached during the experiment by the amino acids.

The locations of the smectite crystals that were altered or even dissolved by E. coli are probably the terminations of the layers at the crystal edges or close to impurities, where the tetrahedral $\mathrm{Si}$ and $\mathrm{Al}$ are not perfectly connected to their neighbors. When present in smectite structures, $\mathrm{Fe}_{(\text {III) }}$ is mostly in octahedral position. It was found here to be enriched in solution with E. coli compared to all solutions with amino acids. Therefore, it may reflect a reduction of $\mathrm{Fe}_{(\text {III) }}$ into $\mathrm{Fe}_{\text {(II) }}$ as reported by several 
studies cited in the review by Mueller [43]. The large release of $\mathrm{Mg}$ by $E$. coli more than by amino acids may reflect dissolution of Mg substituting $\mathrm{Al}$ in octahedral sites. Experimental results of interactions between smectite and bacteria [44] showed that the bacteria enhances both the amount of adsorbed interlayer water and the available pore space, and that interlayered Ca facilitates bacterial attachment to surfaces, while $\mathrm{Fe}_{\text {(III) }}$ favors the production of chelators that enhance the mineral dissolution.

As $\mathrm{Na}$ and $\mathrm{Ca}$ are not constitutive of the smectite crystallographic framework, as well as most $\mathrm{K}$ that might be hosted by but not tied to its structure, these elements are either adsorbed on the crystal surfaces, or hosted as oxides within the crystals. They can also be parts of independent crystals that could be intergrown with the smectite crystals, such as carbonates and salts, as well as organic compounds as just discussed. Their occurrences are, therefore, affected by the amino acids. This could be the case for most trace elements that outline releases of more than about fifty times ( $\mathrm{Rb}, \mathrm{U}, \mathrm{Th}$ and $\mathrm{Pb}$ ), of more than about one hundred times (As), and even up to about one thousand times (Cr, $\mathrm{Co}, \mathrm{Ni}$, and $\mathrm{Zr}$ ), relative to the reference pure water removal when leached by E. coli.

The REEs provide rather specific signatures that differentiate well the impact of the bacteria on the smectite, when associated with given amino acids. Two different patterns were obtained for two types of amino acids when associated or not with E. coli. Glutamic acid with E. coli induces a REE leachate that is to the opposite of that induced by glutamic acid alone: the combined REE distribution shows a lowering of the LREEs and an increasing of the HREEs. The opposite is obtained for the action of alanine alone and of alanine mixed with E. coli (Figure 7). Also to be mentioned are the varied ratios obtained for the REEs extracted by the amino acids relative to that obtained by the pure water leaching: from 50 to 90 for leucine, at about 20 for those by alanine and glutamic acid and below 5 for those by arginine and serine.
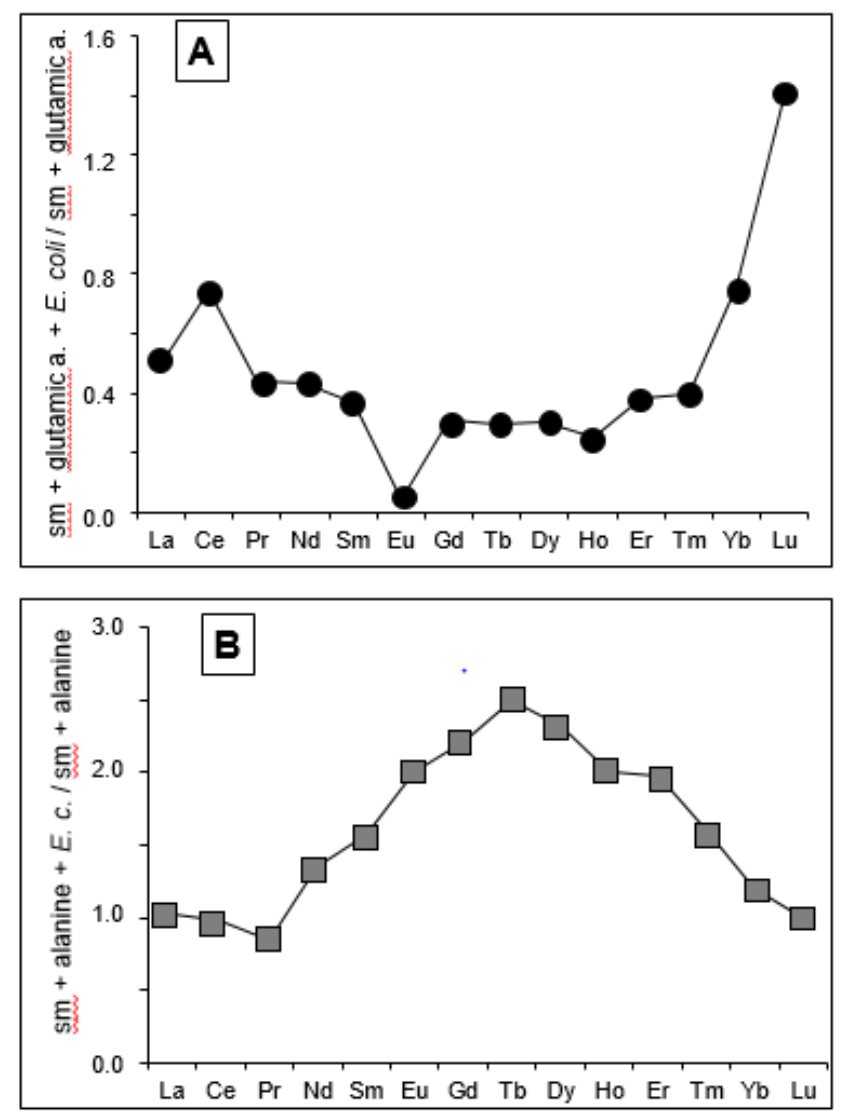

Figure 7: REE distribution patterns in: $(A)$ of a release induced by glutamic acid combined with $E$. coli relative to the release by $E$. coli only, and in (B) of a release by alanine combined with E. coli relative to the release by $E$. coli only. a. stands for acid, c. for coli and sm for smectite.

What impact intensity by the amino acids relative to $E$. coli?

The combination that removes the highest amounts of major elements $(=26.7 \mu \mathrm{g} / \mathrm{g})$ consists of the amino acid's arginine, serine and leucine mixed with the bacteria E. coli (Table 2). The combination removing the second highest amount of major elements $(=23.0 \mu \mathrm{g} / \mathrm{g})$ includes glutamic acid, arginine and $E$. coli. The next highest contents are due to the combination of 
one amino acid at the time with E. coli: successively serine (= $21.4 \mu \mathrm{g} / \mathrm{g})$, glutamic acid $(=19.2 \mu \mathrm{g} / \mathrm{g})$ and alanine $(=17.3 \mu \mathrm{g} / \mathrm{g})$. In trying tri-type combinations, the amount of released elements is decreasing significantly: the mixing of E. coli with glutamic acid, serine and leucine, for instance, only extracts a total of $11.0 \mathrm{mg} / \mathrm{g}$ major elements, which is more than two times less than serine with E. coli.

As stated above, amino acids are also efficient, either individually or combined, in the removal of $\mathrm{Sr}, \mathrm{Ba}, \mathrm{Cr}, \mathrm{Co}, \mathrm{Ni}$ and $\mathrm{Rb}$. Conversely, elements such $\mathrm{As}, \mathrm{Cd}, \mathrm{Pb}, \mathrm{Th}, \mathrm{U}$ and $\mathrm{Zn}$ that are generally harmful to sub-surface environmental systems, are more released from clay material when no amino acid(s) was (were) added to the solution containing $E$. coli. The action of combined amino acids on $\mathrm{Sr}, \mathrm{Ba}, \mathrm{Cr}, \mathrm{Co}, \mathrm{Ni}$ and $\mathrm{Rb}$ indicates that there is more $\mathrm{Sr}$ and $\mathrm{Ba}$ in the solution when serine is combined with leucine, and more than by adding individual amino acids. Chromium is more released in solution with arginine, serine and leucine, and Co in solution with arginine, serine and leucine, but the highest amounts are released with only glutamic acid in the solution. Nickel is also more released with only glutamic acid, while $\mathrm{Rb}$ is more released with glutamic acid combined with arginine.

In summary, this study sketches the roles amino acids may potentially play in soils and geological systems. The effect of individual acids on major elements expressed as a decrease or an increase of the released elements becomes more significant when acids are combined. For instance, elements such as $\mathrm{K}, \mathrm{P}, \mathrm{Mg}, \mathrm{Na}$ and $\mathrm{Ca}$ are more released in solution containing several amino acids, while Fe is more depleted than in solution with only one type, whichever.

\section{Conclusion}

The present experiment was designed to evaluate the impact of the bacteria Escherichia coli combined or not with amino acids (glutamic acid, alanine, arginine, serine and leucine) on crystal dissolution of and elemental desorption from a smectite. The leachates were compared to a basic interaction of the same mineral with pure de-ionized water. Sodium is systematically the dominant released cation, which points to a Na-rich smectite. The bacteria Escherichia coli allowed removal of a Si/Al ratio close to the value of the smectite itself, while far from that released by either the amino acids or by a combination of the bacteria and amino acids. It is, therefore, probable that the bacteria has a dissolution rather than a desorption action. This interpretation is indirectly corroborated by the removal of $\mathrm{Na}$ by the bacteria with a ratio removed $\mathrm{Na}$ /total removed elements much lower than those induced by the amino acids. Also, the leaching impact of the bacteria decreases when mixed with anyone of the selected amino acids.

The study points also to the potential role of amino acids in soils and other geological systems. The high amounts of leached $\mathrm{Si}, \mathrm{Al}, \mathrm{Mn}$ and $\mathrm{Fe}$, which are structural components of clay materials, characterize the batch without any amino acid, while the most released $\mathrm{Ca}$ and $\mathrm{K}$, which are adsorbed elements on smectite-type clay materials, were by the amino acids. Elements such as $\mathrm{Cr}, \mathrm{Ni}$ and $\mathrm{Co}$ are high in solutions with glutamic acid and alanine, while they are low in solutions with arginine and serine. Potassium, $\mathrm{P}, \mathrm{Mg}$, Na and $\mathrm{Ca}$ are more released in solutions containing several amino acids, while if present as oxides they are more depleted than in solutions with only one type of amino acid. Varied distribution patterns of the released rare-earth elements (REEs) allow distinction between the impact of the bacteria producing a progressively decreasing content of the light REEs and a flat distribution for the heavy REEs, and those of the amino acids. The amino acids influence the REE distribution probably along complexation processes due to a discrete occurrence of organic compounds that strongly influence metal sorption on oxide surfaces.

\section{Acknowledgement}

The authors would like to acknowledge Mrs. Munira Al-Habsi of the Biology Department (College of Science, Sultan Qaboos University, Oman) for making available E. coli cultures and Mr. Rabea Al-Maqbali from Department of Animal and Veterinary Sciences (College of Agriculture, Sultan Qaboos University, Oman) for donating the amino acid samples.

\section{References}

1. Schott J, Oelkers EH (1995) Dissolution and crystallization rates of silicate minerals as a function of chemical affinity. Pure and Applied Chemistry 67: 903-910.

2. Kaixuan T, Zheru Z, Zhonggang W (1996) The mechanism of surface chemical kinetics of dissolution of minerals. Chinese Journal of Geochemistry 15(1): 51-60.

3. Colombani J (2008) Measurement of the pure dissolution rate constant of a mineral in water. Geochimica et Cosmochimica Acta 72(23): 56345640 .

4. Sato T, Kuroda M, Yokoyama S, Fukushi K, Nakayama S (2002) Effect of $\mathrm{pH}$ on smectite dissolution rates under alkaline conditions. International Meeting on Clays in Natural and Engineered Barriers for Radioactive Waste Confinement, December 9th, 2002, Reims, France, pp. 11-12.

5. Sato T, Kuroda M, Yokoyama S, Fukushi K, Tanaka T, et al. (2003) Mechanism and kinetics of smectite dissolution under alkaline conditions. Geochimica et Cosmochimica Acta 67: A415.

6. Drever JI, Vance GF (1994) Role of Soil Organic Acids in Mineral Weathering Processes. In: Pittman ED, Lewan MD (Eds.), Organic Acids in Geological Processes. Springer, Berlin, Heidelberg.

7. Sokolova TA (2011) The role of soil biota in the weathering of minerals: A review of literature. Eurasian Soil Science 44(1): 56-72.

8. Ziervogel K, D'souza N, Sweet J, Yan B, Passow U (2014) Natural oil slicks fuel surface water microbial activities in the northern Gulf of Mexico. Front Microbiology 5: 188.

9. Kawano M, Obokata S (2007) The effect of amino acids on the dissolution rates of amorphous silica in near-neutral solution. Clays and Clay Minerals 55(4): 361-368.

10. Kawano M, Hatta T, Hwang J (2009) Enhancement of dissolution rates of amorphous silica by interaction with amino acids in solution at $\mathrm{pH}$ 4. Clays and Clay Minerals 57(2): 161-167. 
11. Ward DB, Brady PV (1998) Effect of Al and organic acids on the surface chemistry of kaolinite. Clays and Clay Minerals 46(4): 453-465.

12. Moyo F, Tandlich R, Wilhelmi BS, Balaz S (2014) Sorption of hydrophobic organic compounds on natural sorbents and organoclays from aqueous and non-aqueous solutions: A mini-review. International Journal of Environmental Research and Public Health 11(5): 50205048.

13. Ehrlich HL (1996) How microbes influence mineral growth and dissolution. Chemical Geology 132(1-4): 5-9.

14. Lloyd JR, Lovely DR (2001) Microbial detoxification of metals and radionuclides. Current Opinion in Biotechnology 12(3): 248-253.

15. Holden JF, Adams MW (2003) Microbe-metal interactions in marine hydrothermal environments. Current Opinion in Chemical Biology 7(2): 160-165.

16. Lloyd JR (2003) Microbial reduction of metals and radionuclides. FEMS Microbiology. Reviews 27(2-3): 411-425.

17. Schröder U, Niessen J, Scholz F (2003) A generation of microbial fue cell with current outputs boosted by more than one order of magnitude. Angewandte Chemistry 115: 2986-2989.

18. Govindaraju K (1994) Compilation of working values and sample description for 383 geostandards. Geostandard Newsletter 18(S1) $1-158$.

19. Samuel J, Rouault R, Besnus Y (1985) Analyse multi-élémentaire standardisée des matériaux géologiques en spectrométrie d'émission par plasma à couplage inductif. Analusis 13: 312-317.

20. Odom IE (1984) Smectite clay minerals: properties and uses. Philosophical Transactions of the Royal Society 311: 1517.

21. Piper DZ, Bau M (2013) Normalized rare earth elements in water, sediments, and wine: Identifying sources and environmental redox conditions. American Journal of Analytical Chemistry 4(10A): 69-83.

22. Vinolas LC, Healey JR, Jones DL (2001) Kinetics of soil microbial uptake of free amino acids. Biology and Fertility of Soils 33(1): 67-74.

23. Vranova V, Zahradnickova H, Janous D, Skene KR, Matharu AS, et al. (2012) The significance of D-amino acids in soil, fate and utilization by microbes and plants: review and identification of knowledge gaps. Plant and Soil 354(1-2): 21-39.

24. Quay SC, Dick TE, Oxender DL (1977) Role of transport system in amino acid metabolism. Leucine toxicity and the branched-chain amino acid transport systems. Journal of Bacteriology 129(3): 1257-1265.

25. Yiling Y, Pollard AM, Höfler C, Poschet G, Wirtz M, et al. (2015) Relation between chemotaxis and consumption of amino acids in bacteria. Molecular Microbiology 96(6): 1272-1282.

26. Koeppenkastrop D, Decarlo EH, Roth M (1991) A method to investigate the interaction of rare earth elements in aqueous solution with metal oxides. Journal of Radioanalytical and Nuclear Chemistry 152(2): 337 346

27. Tiberg C, Sjöstedt C, Gustafsson JP (2018) Metal sorption to spodosol Bs horizons: Organic matter complexes predominate. Chemosphere 196: 556-565.
28. Huang N, Keller WD (1971) Dissolution of clay minerals in dilute organic acids at room temperature. The American Mineralogist 56(5-6): 1082-1095.

29. Lovering TS (1959) Significance of accumulator plants in rock weathering. Geological Society of America Bulletin 70: 781-800.

30. Zysset M, Schindler PW (1996) The proton promoted dissolution kinetics of K-montmorillonite. Geochimica et Cosmochimica Acta 60(6): 921-936.

31. Huertas JF, Chou L, Wollast R (1999) Mechanism of kaolinite dissolution at room temperature and pressure. Part II: Kinetic study. Geochimica et Cosmochimica Acta 63(19-20): 3261-3275.

32. Coppin F, Berger G, Bauer A, Castet S, Loubet M (2002) Sorption of lanthanides on smectite and kaolinite. Chemical Geology 182(1): 57-68.

33. Hradil D, Hostomsky J (2002) Effect of composition and physical properties of natural kaolinitic clays on their strong acid weathering rates. Catena 49(1-2): 171-181.

34. Oelkers E, Schott J, Devidal JD (1994) The effect of aluminum, pH, and chemical affinity on the rates of aluminosilicate dissolution reactions. Geochimica et Cosmochimica Acta 58(9): 2011-2024.

35. Sondia I, Toma V, Filipovi-Vincekovi N (2008) Release of silicon and aluminum from montmorillonite surfaces in aqueous systems. Croatica Chemica Acta 81(4): 623-629.

36. Karnland O (2010) Chemical and mineralogical characterization of the bentonite buffer for the acceptance control procedure in a KBS-3 repository Clay Technology AB September 2010. Technical Report TR10-60. p. 25

37. Abdullahi SL, Audu AA (2017) Comparative analysis on chemical composition of bentonite clays obtained from Ashaka and Tango deposits in Gombe State, Nigeria. ChemSearch Journal 8(2): 35-40.

38. Rollinson H (1993) Using Geochemical Data: Evaluation, Presentation, Interpretation, Pearson Education, New York, NY, USA.

39. Martín-Consuegra SB, Amorós Ortíz-Villajos JA, Pérez-de-los-Reyes C, García Navarro FJ, Ruedas Luna R, et al. (2015) Natural enrichment of trace elements in surface horizons of calcareous soils (La Mancha, Spain). Journal of Chemistry 2015(606837): 10.

40. Chaudhuri S, Clauer N, Semhi K (2007) Plant decay as a major control of river dissolved potassium: A first estimate. Chemical Geology 243(12): 178-190.

41. Čumakov A, Hrnčiarová K, Komadel P (2000) Extraction of potassium from the clay fraction of a bentonite. Ceramics Silikáty 44: 48-53

42. Seppälä A, Puhakka E, Olin M (2016) Effect of layer charge on the crystalline swelling of $\mathrm{Na}+\mathrm{K}+$ and $\mathrm{Ca} 2+$ montmorillonites: DFT and molecular dynamics studies. Clay Minerals 51: 197-211.

43. Mueller B (2015) Experimental interactions between clay minerals and bacteria: A review. Pedosphere 25(6): 799-810.

44. Perdrial JN, Warr L, Perdrial N, Lett MC, Elsass F (2009) Interaction between smectite and bacteria: Implications for bentonite as backfill material in the disposal of nuclear waste. Chemical Geology 264(1-4): 281-294. 


(CC) $\begin{aligned} & \text { This work is licensed under Creative } \\ & \text { Commons Attribution 4.0 License } \\ & \text { DOI: } 10.19080 / I J E S N R .2019 .23 .556103\end{aligned}$

- Quality Editorial servic

- Swift Peer Review

- Reprints availability

- E-prints Service

- Manuscript Podcast for convenient understanding

- Global attainment for your research

- Manuscript accessibility in different formats ( Pdf, E-pub, Full Text, Audio)

- Unceasing customer service

Track the below URL for one-step submission https://juniperpublishers.com/online-submission.php 